\title{
Yap and Taz regulate retinal pigment epithelial cell fate
}

\author{
Joel B. Miesfeld ${ }^{1}$, Gaia Gestri ${ }^{2}$, Brian S. Clark ${ }^{1, *}$, Michael A. Flinn ${ }^{1}$, Richard J. Poole ${ }^{2}$, Jason R. Bader ${ }^{1}$, \\ Joseph C. Besharse ${ }^{1}$, Stephen W. Wilson ${ }^{2}$ and Brian A. Link ${ }^{1, \ddagger}$
}

\begin{abstract}
The optic vesicle comprises a pool of bi-potential progenitor cells from which the retinal pigment epithelium (RPE) and neural retina fates segregate during ocular morphogenesis. Several transcription factors and signaling pathways have been shown to be important for RPE maintenance and differentiation, but an understanding of the initial fate specification and determination of this ocular cell type is lacking. We show that Yap/Taz-Tead activity is necessary and sufficient for optic vesicle progenitors to adopt RPE identity in zebrafish. A Teadresponsive transgene is expressed within the domain of the optic cup from which RPE arises, and Yap immunoreactivity localizes to the nuclei of prospective RPE cells. yap (yap1) mutants lack a subset of RPE cells and/or exhibit coloboma. Loss of RPE in yap mutants is exacerbated in combination with taz (wwtr1) mutant alleles such that, when Yap and Taz are both absent, optic vesicle progenitor cells completely lose their ability to form RPE. The mechanism of Yapdependent RPE cell type determination is reliant on both nuclear localization of Yap and interaction with a Tead co-factor. In contrast to loss of Yap and Taz, overexpression of either protein within optic vesicle progenitors leads to ectopic pigmentation in a dosagedependent manner. Overall, this study identifies Yap and Taz as key early regulators of RPE genesis and provides a mechanistic framework for understanding the congenital ocular defects of Sveinsson's chorioretinal atrophy and congenital retinal coloboma.
\end{abstract}

KEY WORDS: Eye development, Ocular morphogenesis, Zebrafish, Tfec, Hippo signaling, Sveinsson's chorioretinal atrophy, Choroid fissure, Coloboma, Directed differentiation of stem cells

\section{INTRODUCTION}

The neural retina (NR) and retinal pigment epithelium (RPE) arise from a common pool of progenitors during optic vesicle development. Specifically, in fish, cells from the outer layer of the optic vesicle migrate around its margins, as cells within the interior invaginate to form the optic cup (Picker et al., 2009; Kwan et al., 2012; Heermann et al., 2015). Those cells that remain in the outer layer of the optic cup constitute RPE progenitors, whereas the interior is made up of NR progenitors (Picker et al., 2009; Kwan et al., 2012; Heermann et al., 2015). These two retinal populations are distinguishable long before overt functional differentiation, as

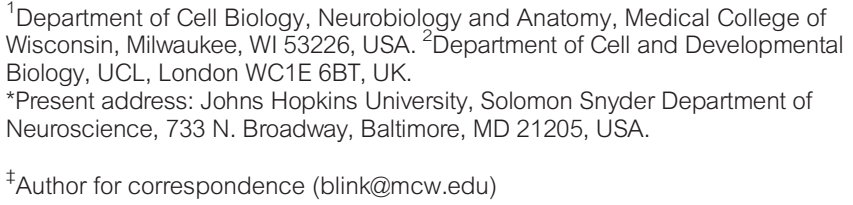

${ }^{\ddagger}$ Author for correspondence (blink@mcw.edu)

This is an Open Access article distributed under the terms of the Creative Commons Attribution License (http://creativecommons.org/licenses/by/3.0), which permits unrestricted use, distribution and reproduction in any medium provided that the original work is properly attributed.

Received 22 October 2014; Accepted 10 July 2015 the inner elongated cells of the NR are morphologically distinct from the outer flattened prospective RPE cells.

Initially, optic vesicle progenitors express the same transcription factor-encoding genes (such as $\ln x 2$, pax6, six $3, v s \times 2$ and $r \times 1 / 2 / 3$ ), and signaling from surrounding tissues subsequently contributes to the regionalization of the optic vesicle into prospective RPE and NR domains (eye morphogenesis is reviewed in supplementary material Movie 1; Sinn and Wittbrodt, 2013; Fuhrmann et al., 2014). Even after RPE and NR domains are established, both cell populations maintain the ability to transdifferentiate (Sinn and Wittbrodt, 2013; Fuhrmann et al., 2014). As eye development progresses, multiple signaling pathways (including BMP, FGF, Notch, WNT, SHH and TGF $\beta$ ) continue to influence the expression of transcription factors that function in the differentiation and maintenance of the NR and RPE (Sinn and Wittbrodt, 2013; Fuhrmann et al., 2014). Although several transcriptional modulators (including Mitf, Otx and $\beta$-catenin) have been implicated in RPE differentiation and maintenance, none has yet been shown to mediate the initial specification of RPE cell identity. Therefore, either combinations of known transcriptional regulators or novel factors must initiate specification of the RPE. Here we provide evidence that targets of the Hippo signaling pathway are key regulators of RPE specification.

The Hippo kinase signaling cascade widely regulates apoptosis, proliferation and cell fate decisions during development by controlling the localization and stability of the transcriptional coactivators Yes-associated protein 1 (Yap1, or more commonly Yap) and WW domain containing transcription regulator 1 (Wwtr1, or more commonly Taz) (Varelas, 2014). Inactive Hippo signaling results in nuclear localized Yap and Taz and an increase in transcription of Yap/Taz target genes. The main nuclear binding partners for Yap and Taz are the Tea domain (Tead) transcription factors. There are four Tead homologs in vertebrates, which together are broadly expressed across tissues during development (Mann et al., 2007; Naye et al., 2007).

An involvement of Yap and Tead in eye development is suggested by the prominent expression of a Yap/Taz-Teadresponsive transgene in tissues and cells undergoing complex morphogenetic movements, including the eyes (Miesfeld and Link, 2014). Furthermore, heterozygous loss-of-function mutations in YAP1 in humans can result in autosomal dominant coloboma and a mutation within the Yap-binding domain of TEAD1 causes Sveinsson's chorioretinal atrophy (SCRA), an autosomal dominant loss of RPE, choroid, and photoreceptors radiating from the optic nerve head (Fossdal et al., 2004; Williamson et al., 2014). Although these mutations and associated diseases have been described, the mechanism(s) underlying the defects is unknown.

In this study we address the roles of Hippo signaling components during zebrafish eye development. We analyzed loss-of-function mutations in both yap and taz, transgenic lines that manipulate Yap and Taz activity in a tissue-specific manner, and reporter lines that 
label RPE progenitors. These tools revealed roles for the Yap and Taz transcriptional co-activators in choroid fissure closure and RPE specification that are likely to be conserved between zebrafish and humans.

\section{RESULTS}

\section{Yap/Taz-Tead signaling is active during optic cup} morphogenesis

Analysis of the $4 x$ GTIIC:d2GFP transgenic line (Miesfeld and Link, 2014) suggested a role for Yap/Taz-Tead activity in the developing lens, NR and RPE (Fig. 1; supplementary material Movie 2). Reporter transgene expression was evident in the ectoderm overlying the optic vesicle at $14 \mathrm{~h}$ post-fertilization (hpf) $\left(\right.$ Fig. $\left.1 \mathrm{~A}^{\prime}\right)$ and in the lens placode and presumptive RPE by 18 hpf (Fig. 1B'). Throughout optic cup invagination transgene activity was present at low levels in NR progenitors and more prominently in RPE progenitors (Fig. 1; supplementary material Movie 2). Fluorescence is first evident in the optic cup midway through its morphogenesis, but Yap/Taz-Tead activity is likely to initiate earlier than this given the delay between transcription of the transgene and fluorescence of its protein product. To investigate the roles of Yap and Taz, we generated mutations within each gene and analyzed the consequences for eye formation.

\section{yap mutants lack RPE cells}

yap mutant alleles were generated using transcription activator-like effector nuclease (TALEN) technology. Multiple founders containing
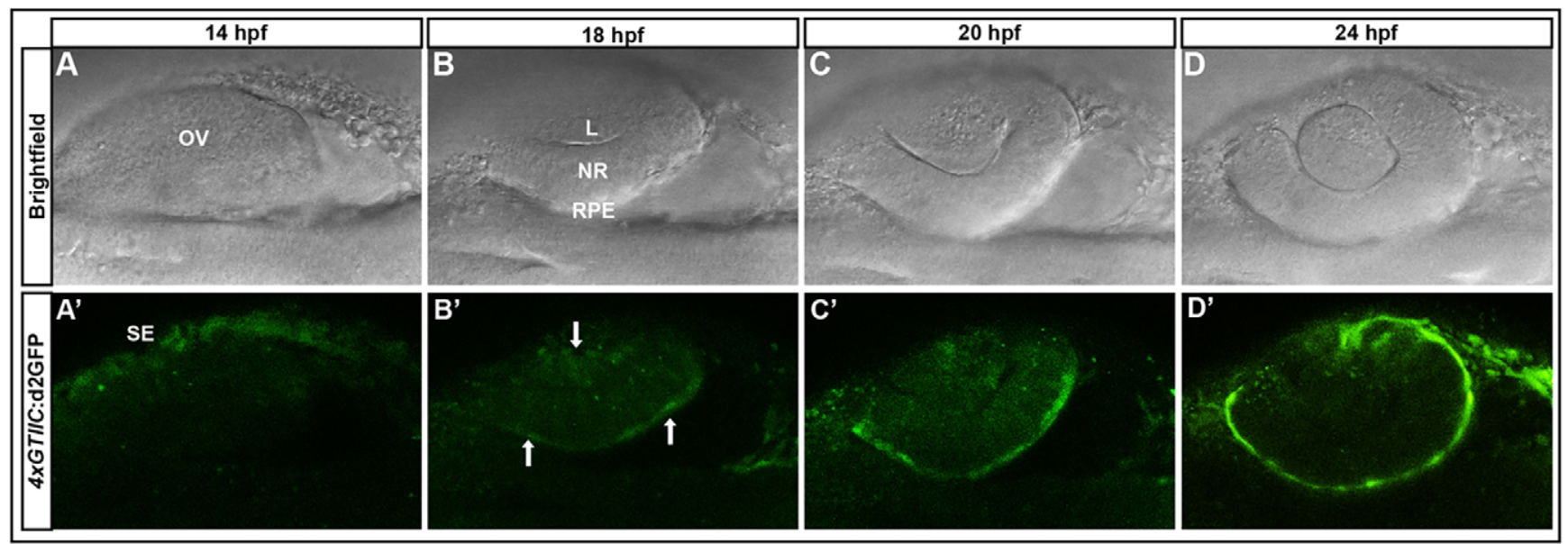

E Yap

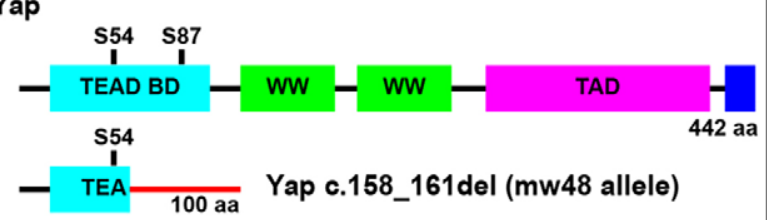

Taz

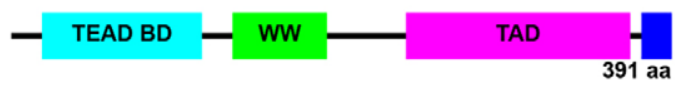

_ TEA_ 134 aa Taz c.156_160del (mw49 allele)
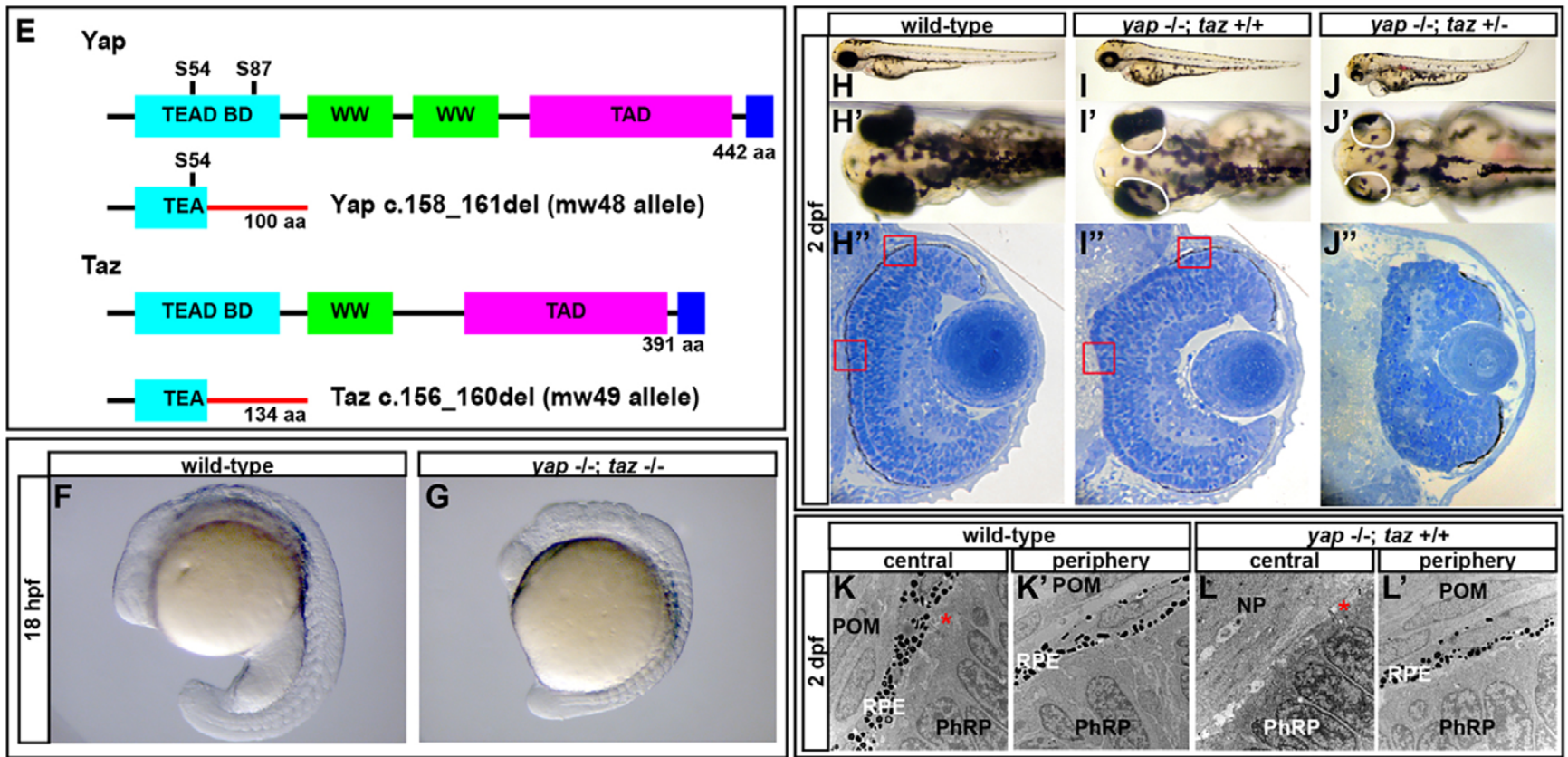

Fig. 1. A Yap/Taz-Tead reporter transgene is dynamically expressed during optic cup morphogenesis and yap ${ }^{-1-}$ mutants exhibit RPE defects. $\left(A-D^{\prime}\right)$ Images of live zebrafish from 14-24 hpf showing optic cup development and 4xGTIIC:d2GFP transgene expression (green). Arrows indicate cells that are expressing the transgene while undergoing morphogenesis. (E) Schematic of wild-type and mutant Yap and Taz. Yap S54 is an essential residue for Tead binding and S87 is phosphorylated by Lats leading to cytoplasmic retention. The Yap c.158_161del and Taz c.156_160del mutants contain frameshifts resulting in early stop codons. TEAD BD, Tead transcription factor binding domain (light blue); WW, dual tryptophan motif (green); TAD, transactivation domain (fushia); PDZ domain (dark blue). $(\mathrm{F}, \mathrm{G}) \mathrm{yap}^{-/-} ; \mathrm{taz}^{-/-}$embryos arrest by $18 \mathrm{hpf}$ with multiple defects. $\left(\mathrm{H}-\mathrm{J}^{\prime \prime}\right)$ Live embryos $\left(\mathrm{H}-\mathrm{J}^{\prime}\right)$ and sections $\left(\mathrm{H}^{\prime \prime}, \mathrm{I}^{\prime \prime}, \mathrm{J}^{\prime \prime}\right)$ of yap ${ }^{-/-} ; \operatorname{taz}^{+/+}\left(\mathrm{I}-\mathrm{I}^{\prime \prime}\right)$ and $y a p^{-1-} ; \operatorname{taz}^{+/-}\left(\mathrm{J}-\mathrm{J}^{\prime \prime}\right)$ showing RPE defects and additional NR defects in yap ${ }^{-1-} ; \operatorname{taz}^{+/-}$mutants $\left(\mathrm{J}^{\prime}\right)$ compared with control $\left(\mathrm{H}-\mathrm{H}^{\prime \prime}\right)$. Boxed areas indicate locations of TEM analysis. (K-L') Transmission electron microscopy analysis showing areas of normal RPE development $\left(\mathrm{L}^{\prime}\right)$ and areas devoid of RPE (L) in yap ${ }^{-1-}$ eyes. Asterisk indicates the presence of primary cilia on neuroepithelial cells. L, lens; OV, optic vesicle; NR, neural retina; RPE, retinal pigment epithelium; SE, surface ectoderm; POM, periocular mesenchyme; NP, neuropil; PhRP, photoreceptor progenitors. 
different insertion or deletion alleles were obtained and two lines established. A 4 nt deletion, yap c.158_161del $\left(y_{a p}{ }^{m w 48 / m w 48}\right.$, referred to as $y a p^{-/-}$), resulted in a frameshift within the Teadbinding domain of Yap leading to a predicted incorrect amino acid sequence and an early stop codon (Fig. 1E). $y_{a p^{-/-}}$embryos had a 3fold decrease in yap mRNA $(P=0.0009)$ and lacked Yap immunoreactivity (Fig. 3A-C), suggesting nonsense-mediated mRNA decay and the absence of Yap protein.

By 1 day post-fertilization (dpf), yap ${ }^{-/-}$mutants show mild heart edema, vascular hemorrhages and an impairment in RPE development (Fig. 1I-I",L; supplementary material Fig. S1; data not shown). Some $\mathrm{yap}^{-/}$fish survived to adulthood and none of the early phenotypes were exacerbated through the loss of maternal Yap contribution in embryos generated from $\mathrm{yap}^{-/-}$mothers. Embryos heterozygous for the $m w 48$ or other mutant alleles described here appeared overtly normal.

The loss of RPE in $y a p^{-/-}$mutants is noticeable as soon as melanization begins and becomes more apparent once retinal pigmentation is complete (Fig. $1 \mathrm{I}^{\prime}, \mathrm{I}^{\prime \prime}$; supplementary material Fig. S1). RPE deficiency typically occurs at the back of the eye but can also variably occur on the lateral and ventral surfaces and can differ in phenotypic extent between eyes of the same embryo. Electron microscopy of 2 dpf $y_{a p}{ }^{-/}$eyes revealed normal RPE cells in regions with visible pigmentation (Fig. 1L'). However, in areas lacking pigmentation there was an absence of flattened cells characteristic of either RPE or periocular mesenchyme, and NR progenitors directly abutted the forebrain neuropil (Fig. 1L). The retinal neuroepithelia appeared normal, possessed the modified primary cilia that form photoreceptor outer segments, and displayed proper retinal layering, even beneath regions lacking RPE (Fig. 1I").

\section{yap mutants exhibit variable phenotypes including coloboma}

Although fully penetrant, the RPE phenotype in $\mathrm{app}^{-/-}$mutants was variable and other phenotypes, including viability, showed similar variability. Additional support for phenotypic variability in yap mutants came from assessment of another allele, nl13, which exhibited a colobomatous phenotype (Fig. 2G-H) and was identified through a forward genetic screen of ENU-induced mutations. The nl13 mutation was localized between Zv2560 and Zv8353 on chromosome 18 using bulked segregant analysis with SSLPs. yap lies within this interval and, given that mutations in human YAP1
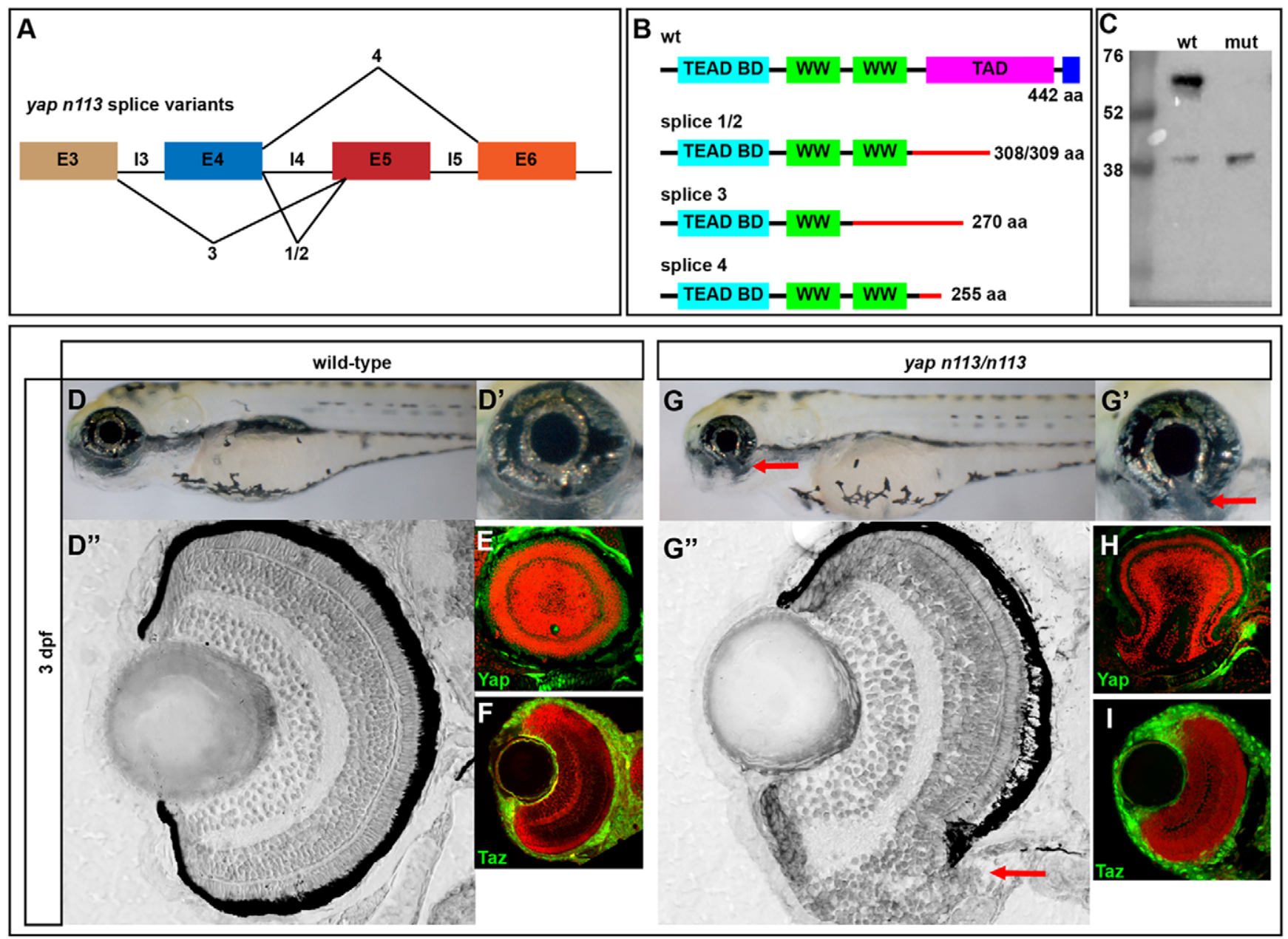

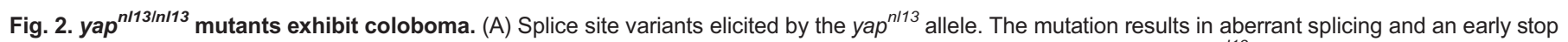

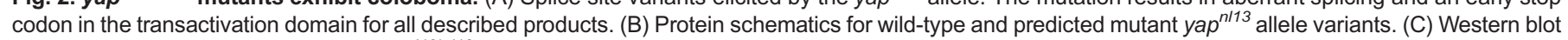

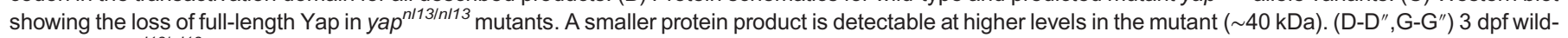

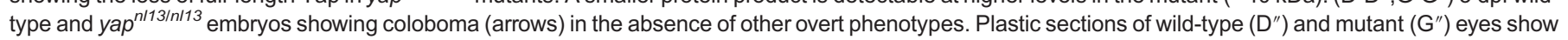
the coloboma (arrow) phenotype and RPE deficits that are sometimes observed in the ventral retina. (E,F,H,I) Sections showing Yap and Taz proteins (green) in wild-type and yap ${ }^{\text {n/13/n/13 }}$ mutant eyes. Red counterstain (TO-PRO3) shows nuclei. 

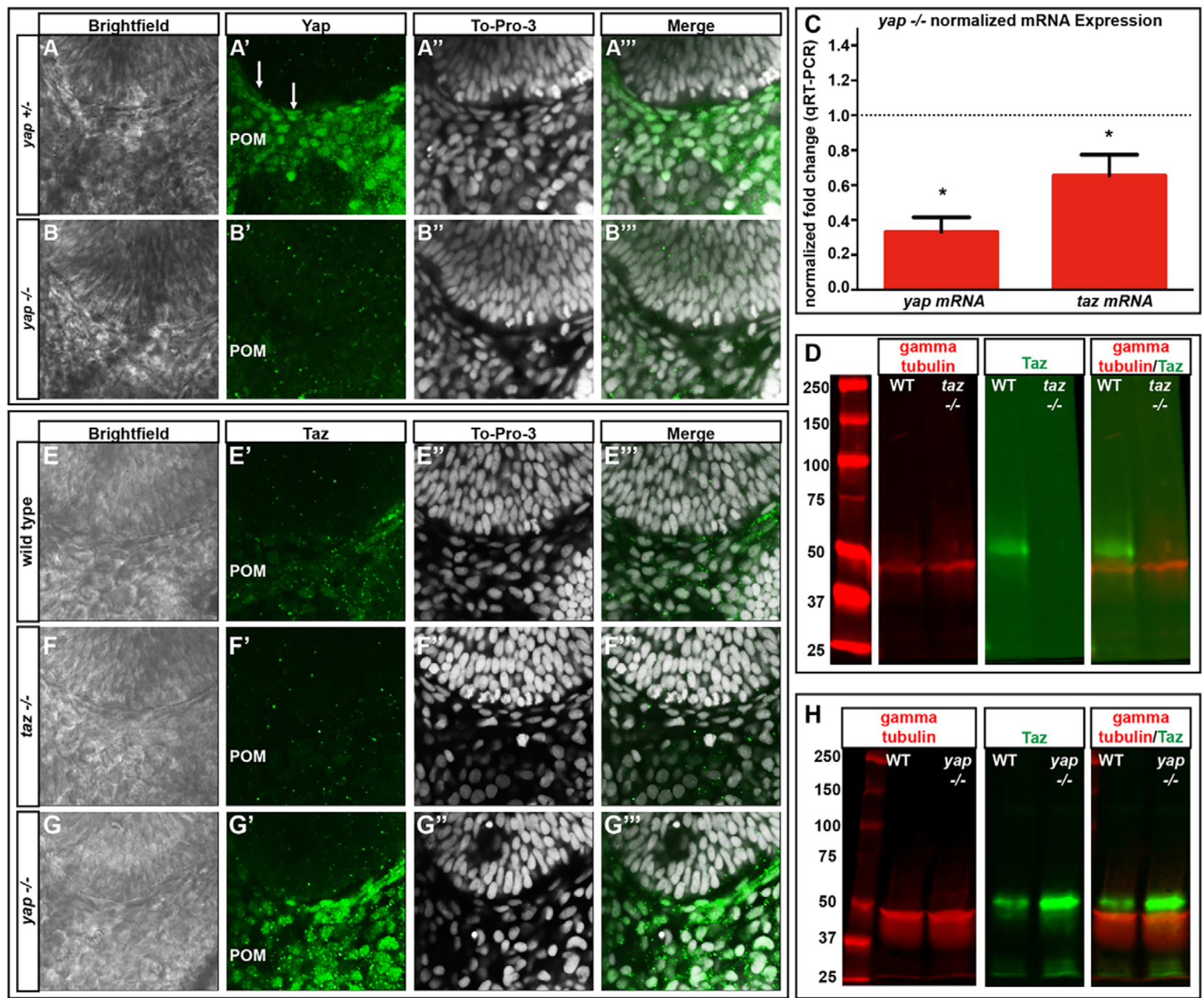

Fig. 3. yap mRNA and Yap protein levels are decreased and Taz protein increased in yap ${ }^{-1-}$ embryos. (A-B"') Yap immunoreactivity in wild-type and yap

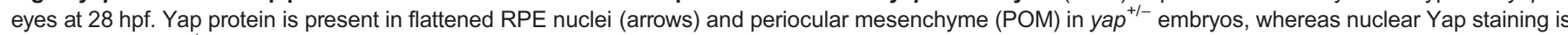
absent in the $y a p^{-1-}$ mutant. (C) qRT-PCR analysis of whole embryos at $32 \mathrm{hpf}$ showing a decrease in yap (3-fold, $\left.{ }^{*} P=0.0002\right)$ and taz (1.5-fold, ${ }^{*} P=0.0270$ ) mRNA in yap ${ }^{-1-}$ mutants. Dotted line indicates normalized expression levels of yap and taz in wild-type embryos. An unpaired $t$-test was performed and statistical significance determined using the Holm-Sidak method. Error bars represent s.e.m. (D) Western blot showing Taz protein ( $52 \mathrm{kDa})$ in wild-type and its absence in taz $^{-/-}$adult heart tissue. (E-G") Taz immunoreactivity in wild-type, taz $^{-/-}$and yap ${ }^{-/-}$embryos at 28 hpf. $(\mathrm{H})$ Western blot of Taz protein from $2 \mathrm{dpf}$ wild-type ( $\left.n=20\right)$ and yap ${ }^{-1-}$ mutant $(n=20)$ whole embryos.

can lead to isolated and syndromic coloboma (Williamson et al., 2014), this gene was a good candidate for harboring the mutation. The genomic mutation was identified as a single base change from A to $\mathrm{T}$ in the splice acceptor site of intron 4 . Sequencing the coding region from mutant cDNA revealed four splice variants (Fig. 2A,B), with the main isoform resulting in a deletion of $11 \mathrm{nt}$ between positions 673 and 684, generating a stop codon at amino acid 309, the beginning of the transactivation domain (Fig. 2A,B). Yap immunoreactivity was still detected in yap ${ }^{n l 13 / n l 13}$ mutants (Fig. 2E,H) but western blots showed the presence of a smaller than wild-type protein ( $\sim 40 \mathrm{kDa}$ versus $\sim 65 \mathrm{kDa}$; Fig. $2 \mathrm{C}$ ).

To test whether the mutation in yap caused the coloboma phenotypes, we injected synthetic yap-GFP RNA into embryos from a cross between carriers and assessed phenotypic rescue. In non-injected controls, $25.45 \% \quad(n=28 / 110)$ of embryos showed coloboma, whereas injection of $200 \mathrm{pg}$ wild-type yap mRNA rescued the coloboma phenotype ( $n=106 / 110$ normal; 4/110 showed coloboma). As in humans with YAPl mutations, the coloboma phenotype in yap nl13/nl13 zebrafish embryos could be either unilateral or bilaterally symmetric. RPE deficits were observed in yap ${ }^{n l 13 / n l 13}$ embryos as in other alleles, but these were restricted to the ventral eye around the open choroid fissure. Also, as in other alleles, NR lamination was generally normal although outer retinal neurons were significantly disrupted where the coloboma was present (Fig. 2G-G"). Other than the ventral eye phenotypes, yap ${ }^{\text {nl13/nll3 }}$ embryos showed no overt phenotypes (Fig. 2G).

\section{taz mutant alleles enhance the yap $^{-l-}$ phenotype}

The variable loss of RPE in $y a p^{-/-}$embryos (and in other alleles) can be rescued by raising embryos at $20.5^{\circ} \mathrm{C}$ (supplementary material Table S1). Together with the observation that some RPE develops in mutants, this suggests that another factor(s) contributes 
to RPE development. An obvious candidate is Taz, a homologous transcriptional co-regulator, and so we generated a mutant allele with a $5 \mathrm{nt}$ deletion, taz c.156_160del ( $\operatorname{taz}^{m w 49}$, referred to as $\mathrm{taz}^{-1-}$ ). This mutation leads to a frameshift deletion in the Teadbinding domain of Taz and a resultant truncated protein containing a sequence of 82 incorrect amino acids and an early stop (Fig. 1E). Despite lacking detectable Taz protein (Fig. 3D), $\mathrm{taz}^{-/-}$embryos have no overt embryonic phenotype and survive to adulthood.

Although $\mathrm{taz}^{-/-}$RPE appears normal, the inclusion of one mutant taz allele within a $\mathrm{yap}^{-/-}$background $\left(\mathrm{yap}^{-/-} ; \mathrm{taz}^{+/-}\right)$enhanced the loss of RPE and led to more severe body axis, heart and NR defects compared with $\mathrm{yap}^{-/}$siblings (Fig. 1J-J"). Double-homozygous mutant $\left(\mathrm{yap}^{-/-} ; \mathrm{taz}^{-/-}\right)$embryos arrest before eye morphogenesis is complete, precluding assessment of RPE development (Fig. 1F,G).

Unlike in $\mathrm{yap}^{-/-} ; \mathrm{taz}^{+/+}$embryos, rearing $\mathrm{yap}^{-/-} ; \mathrm{taz}^{+/-}$embryos at low temperatures did not rescue the RPE phenotype (supplementary material Table S2), suggesting that the temperature sensitivity of the $y a p^{-1-}$ phenotype is due to redundancy with Taz (as also described in other situations; Nishioka et al., 2009). Analysis of taz mRNA levels within yap ${ }^{-/}$ embryos did not reveal compensatory changes in transcript abundance (Fig. 3C). However, Taz immunoreactivity appeared increased at $28 \mathrm{hpf}$ (Fig. 3G-G'"'), higher total Taz protein levels were detected in $2 \mathrm{dpf} \mathrm{yap}^{-/-}$embryos (Fig. 3H) and nuclear localization appeared enhanced in yap nl13/nl13 mutants (Fig. 2F,I).

\section{Nuclear activity of Yap/Taz-Tead is required for RPE genesis}

SCRA is an autosomal dominant congenital disorder characterized by loss of RPE and photoreceptors proximal to the optic nerve head (Fossdal et al., 2004; Jonasson et al., 2007) and is caused by a tyrosine-to-histidine mutation in the Yap-binding domain of TEAD1 (Kitagawa, 2007). yap ${ }^{-/-}$embryos share phenotypic features of SCRA patients with TEAD1 deficiencies, suggesting that the consequences of Yap deficiency are mediated through Tead.

In order to determine if a lack of Yap-Tead activity is responsible for RPE loss, we first tested whether the Yap and Tead binding domains are conserved in zebrafish. We generated plasmids encoding wild-type Tead1a, a mutant version equivalent to the YAP1 binding-deficient allele of SCRA patients (Tead1a Y417H), wild-type Yap, and a variant with a mutation within the putative Tead-binding domain (Yap S54A) (Zhao et al., 2008; Chen et al., 2010) (Fig. 4A). Consistent with studies in other species, transfection assays in HEK293 cells showed that zebrafish Yap and Teadla are able to interact with each other, whereas the mutant variants Yap S54A and Teadla Y417H are unable to bind (Fig. 4B). Additionally, overexpression of the autosomal dominant Teadla Y417H allele within the optic vesicle resulted in RPE loss around the optic nerve, similar to observations in SCRA patients (Fig. 4C-E').

To further investigate the role of Tead in Yap-mediated RPE genesis, we generated a Tead domain-specific yap mutant allele.
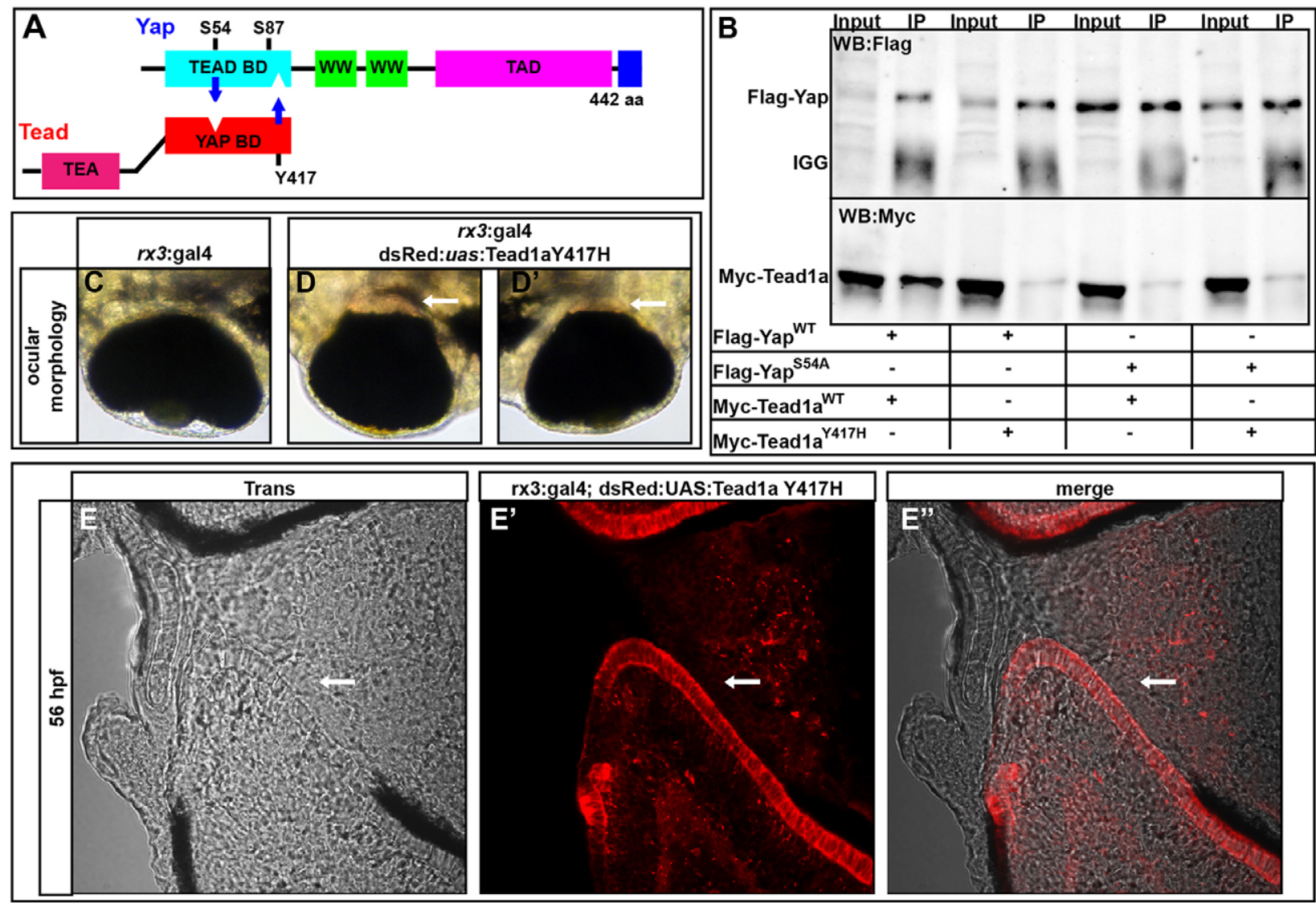

Fig. 4. Yap and Tead1a zebrafish protein interactions are conserved. (A) Schematic of the zebrafish Yap and Tead binding domain (BD) interaction sites. (B) Immunoprecipitation of zebrafish Yap and Tead1a wild-type and Tead-binding-deficient Yap (Yap S54A) and Yap-binding-deficient Tead1a (Tead1a Y417H) isoforms. All the mutated protein variants lose the ability to interact, in contrast to the wild-type proteins. Immunoprecipitation (IP) was with an anti-Flag antibody. $\left(C-E^{\prime \prime}\right)$ Whole eyes (C-D') and sections (E-E") showing RPE loss surrounding the optic nerve head after overexpression of Tead1a Y417H. Photoreceptors are red owing to late expression of the $r \times 3: G a l 4$ driver in these cells. Arrows indicate areas lacking RPE. 
A c. $158 \_178 \mathrm{del}\left(y a p^{m w 69 / m w 69}\right.$, referred to as $\left.y a p^{\Delta T B / \triangle T B}\right)$ deletion in yap created a seven amino acid in-frame deletion that eliminates the critical S54 and surrounding amino acids (Chen et al., 2010) within the Tead-binding domain (Fig. 5A). yap ${ }^{\Delta T B / \Delta T B}$ mutants had slightly lower levels of yap mRNA (Fig. 5E). Although Yap protein was still detected (Fig. 5F-G'") in yap ${ }^{\Delta T B / \triangle T B}$ embryos, loss of RPE was still evident (Fig. 5B-D).

Additional evidence for a nuclear role of Yap in RPE genesis came from analysis of the consequences of overexpression of dominant-negative forms of Yap and Taz that contain nuclear localization sequences but lack transactivation domains (NLSYapDN, NLS-TazDN) (Cao et al., 2008; Miesfeld and Link, 2014). When either dominant-negative Yap or Taz was mosaically overexpressed in RPE/retinal precursors using vsx2:Gal4 (Miesfeld and Link, 2014) or $r x 3:$ Gal4, loss of RPE was observed (Fig. 8E-G; supplementary material Fig. S2). By contrast, expression of either dominant-negative protein in periocular mesenchymal cells using a foxclb:Gal4 driver did not result in RPE defects (not shown). Collectively, these results suggest that Yap/Taz-Tead-dependent transcription is required autonomously within optic cup progenitor cells to generate RPE.

\section{Optic vesicle proliferation and apoptosis are unaffected in yap $^{-I-}$ mutants}

Two well-reported functions of the Yap/Taz-Tead interaction are the control of cell proliferation and death. However, there was no obvious difference in proliferation or cell death in $y_{a p}{ }^{-/}$eyes at $14 \mathrm{hpf}$, before markers of pigmentation are detected, at $18 \mathrm{hpf}$, the onset of observed Yap/Taz-Tead reporter transgene activity, or at $24 \mathrm{hpf}$, when RPE loss is first detected (Fig. 6A,B). To more specifically assess the proliferation of prospective RPE cells, we used a $-2.7 \mathrm{~kb}$ $t f e c$ :eGFP transgene that is expressed (as is the tfec gene; Lister et al., 2011) in presumptive and definitive RPE cells (Fig. 6C-E"'; supplementary material Movies 3 and 4). When analyzed at $14 \mathrm{hpf}$, there was no difference in the numbers of proliferating $-2.7 \mathrm{~kb} \mathrm{tfec}$ : eGFP-positive cells between $\mathrm{yap}^{-/-}$and sibling controls (Fig. 6F).

To further test the potential significance of cell death and proliferation as contributors to the mutant phenotype, each process was inhibited during optic cup morphogenesis. To ameliorate cell death, yap mutants and their wild-type siblings were injected with tp53 morpholino (Robu et al., 2007) and bcl-xl mRNA (Sidi et al., 2008). Although normal apoptosis in the lens epithelium was inhibited in all embryos, the yap mutant RPE phenotype was not
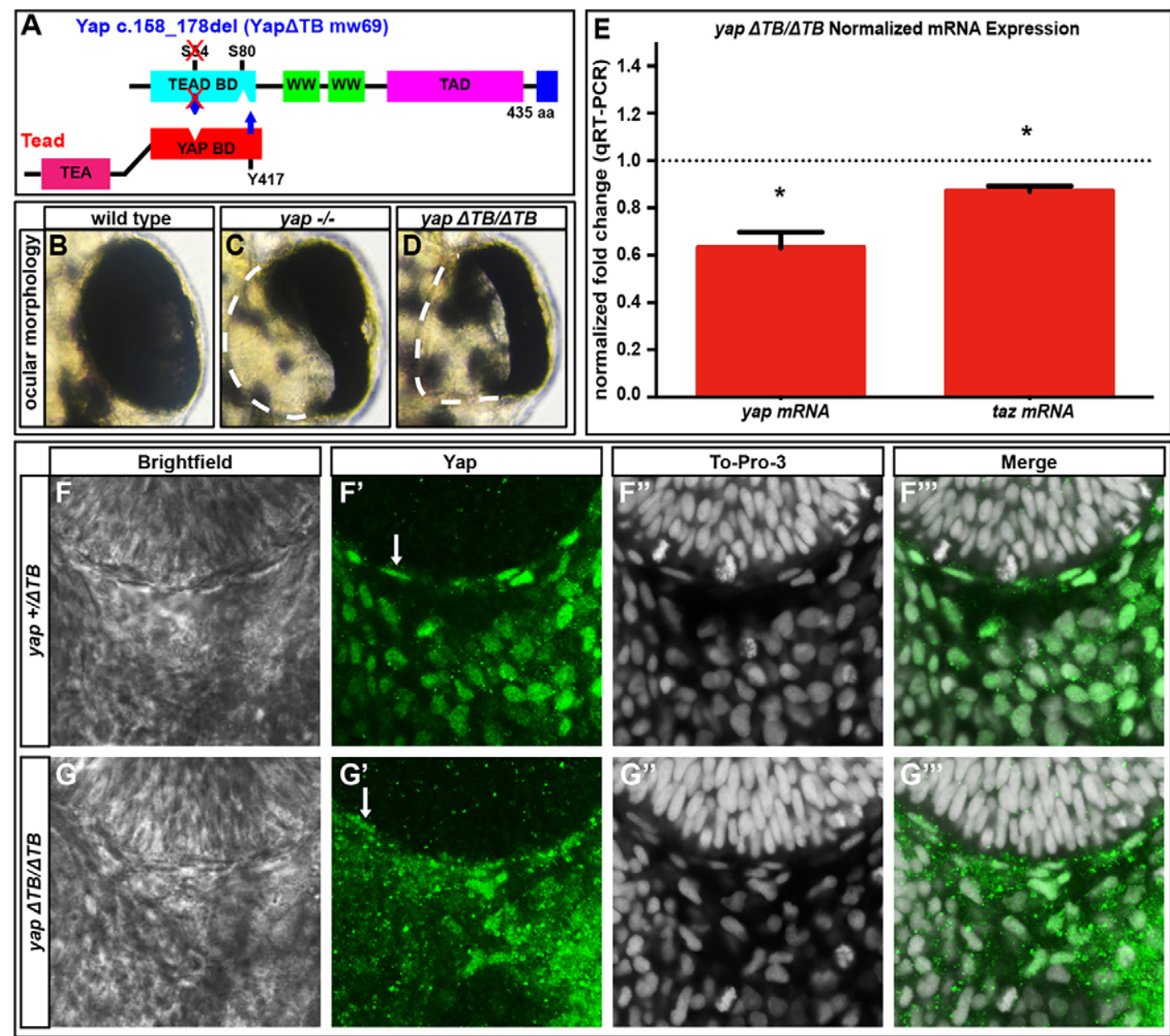

Fig. 5. Tead-binding-deficient yap ${ }^{\Delta T B L T B}$ mutants lack RPE but maintain yap mRNA and Yap protein levels. (A) The Tead-binding-deficient yap ${ }^{\triangle T B / \Delta T B}$

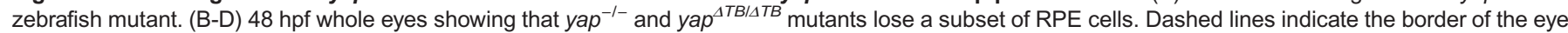
(E) qRT-PCR analysis of whole embryos at $32 \mathrm{hpf}$ revealing a decrease in yap (1.6-fold, ${ }^{*} P=0.0052$ ) and taz (1.15-fold, $\left.{ }^{*} P=0.0038\right) \mathrm{mRNA}$ in yap ${ }^{\Delta T B / \Delta T B}$ mutants. Dotted line indicates the normalized expression levels of yap and taz in wild-type embryos. An unpaired $t$-test was performed and statistical significance was determined using the Holm-Sidak method. Error bars indicate s.e.m. (F-G'") Yap protein expression in yap ${ }^{+/ \Delta T B}\left(\mathrm{~F}-\mathrm{F}^{\prime \prime \prime}\right)$ and yap ${ }^{\Delta T B / \Delta T B}\left(\mathrm{G}-\mathrm{G}^{\prime \prime \prime}\right)$ at $28 \mathrm{hpf}^{\prime}$ Yap protein is present in flattened RPE nuclei (arrows) and periocular cells in yap $p^{+\Delta T B}$ and yap ${ }^{\Delta T B / \Delta T B}$ embryos. 


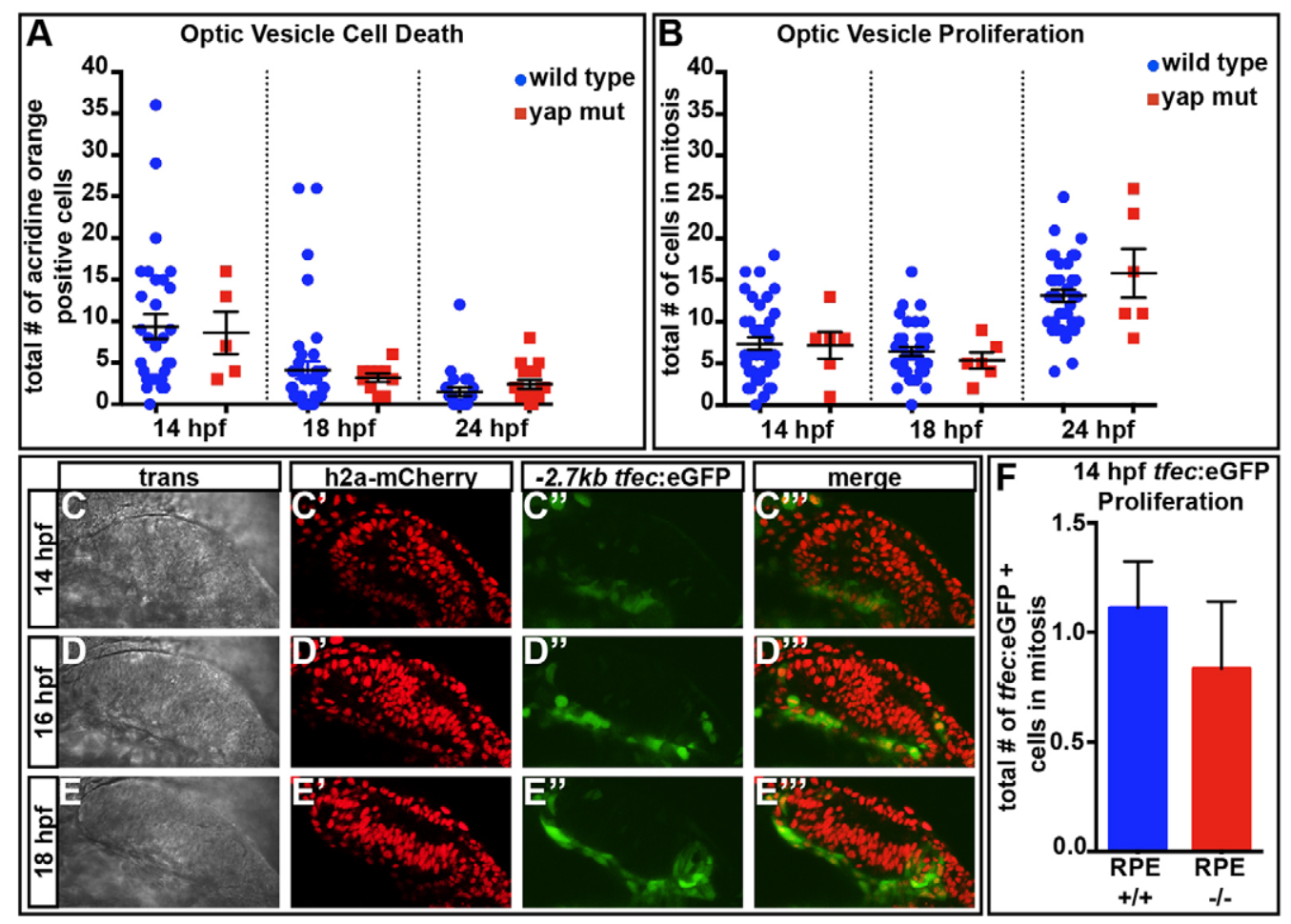

Fig. 6. Cell death and proliferation are normal in yap ${ }^{-1-}$ eyes. (A) The numbers of dying cells identified by Acridine Orange staining do not differ in yap ${ }^{-/-}$mutants at $14(P=0.8465)$, $18(P=0.6542)$ or $24(P=0.2558) \mathrm{hpf}$ as compared with wild type. Numbers of eyes analyzed: $14 \mathrm{hpf}$, wt $n=31$, yap $^{-/-} n=5 ; 18 \mathrm{hpf}$, wt $n=39$, yap $^{-1-}$ $n=10 ; 24$ hpf, wt $n=24$, yap $^{-1-} n=16$. (B) Eye field mitotic cell counts do not differ between yap ${ }^{+/ ?}(\mathrm{wt})$ and $y a p^{-1}$ at $14(P=0.9205), 18(P=0.4329)$ and $24(P=0.2222)$ hpf. Numbers of eyes analyzed: $14 \mathrm{hpf}$, wt $n=38$, yap $^{-/}$ $n=6 ; 18 \mathrm{hpf}$, wt $n=38$, yap $^{-1-} n=6$; $24 \mathrm{hpf}$, wt $n=36$, yap ${ }^{-l-} n=6$. (C-E"') Time-course of expression of tfec:eGFP in prospective RPE cells. (F) Mitotic cell counts of tfec:eGFP ${ }^{+}$ cells do not differ between wild-type and yap $^{-1-}$ eyes at $14 \mathrm{hpf}$ $(P=0.5408)$. Numbers of eyes analyzed: wt $n=18$, yap $^{-1-} n=6$. $P$ values were obtained using an unpaired $t$-test with equal s.d. Error bars indicate s.e.m. Wild type included full RPE coverage, whereas yap $^{-1-}$ showed some RPE loss. rescued (not shown). Additionally, impairing proliferation in wildtype embryos from 10.5 to $26 \mathrm{hpf}$ with hydroxyurea and aphidicolin did not phenocopy the RPE loss seen in yap mutants (not shown). These results suggest that neither changes to proliferation nor cell death are major contributing factors to RPE loss and, therefore, that Yap activity may impart RPE identity upon bi-potential optic vesicle cells.

\section{Yap and Taz are essential for the generation of RPE}

Our results suggest that Yap/Taz function might be essential for cells to adopt RPE identity; however, our genetic analyses did not allow us to definitively show this because $\mathrm{yap}^{-/-} ; \mathrm{taz}^{-/-}$embryos arrest before RPE begins to pigment. Consequently, to test whether retinal precursors lacking Yap and Taz could form RPE, we transplanted fluorescently labeled yap; taz mutant cells into albino embryos that carried wild-type yap and taz alleles. Albino embryos can generate xanthophores, iridophores and leucophores but not the melanin pigment found in RPE cells (Streisinger et al., 1986; Tsetskhladze et al., 2012). Therefore, we could easily assess whether the fluorescently labeled optic vesicle cells of various yap; taz genotypes were able to generate RPE (Fig. 7A-C'). We scored the presence/absence of pigmented RPE in eyes that contained clones of transplanted cells in the NR to ensure that transplanted mutant cells were properly targeted to the eye field of the host blastula/gastrula.

In mosaic analyses, cells of all genotypes contributed to both NR and RPE, except for $y a p^{-/-} ; t^{-/-}$, which only contributed to NR

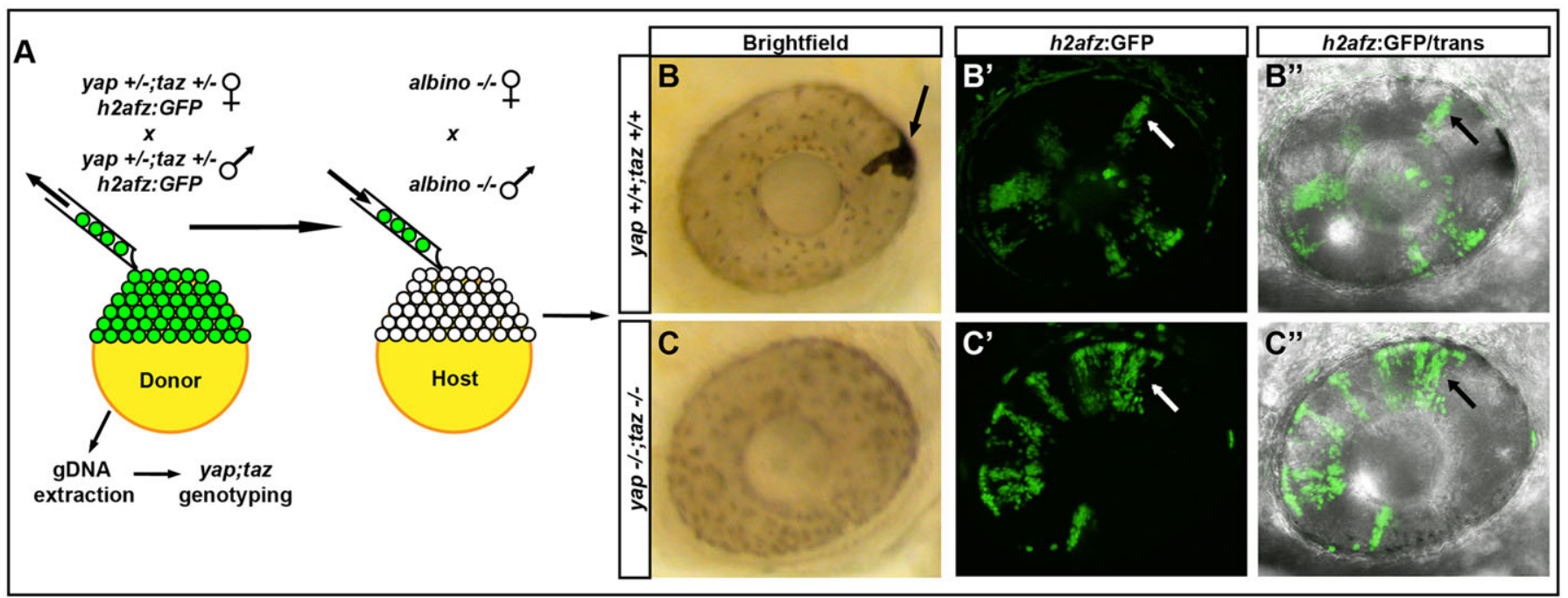

Fig. 7. yap $^{-1-} ;$ taz $^{-l-}$ transplanted cells do not contribute to the RPE. (A) Methods used to analyze the contribution of transplanted cells to the RPE and NR.

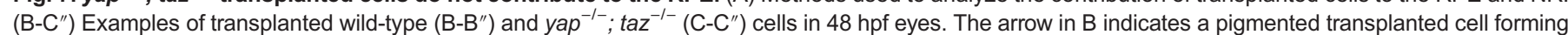
$\mathrm{RPE}$ in the albino host; other black/white arrows indicate $\mathrm{H} 2 \mathrm{a}-\mathrm{GFP}^{+}$clones in NR. 

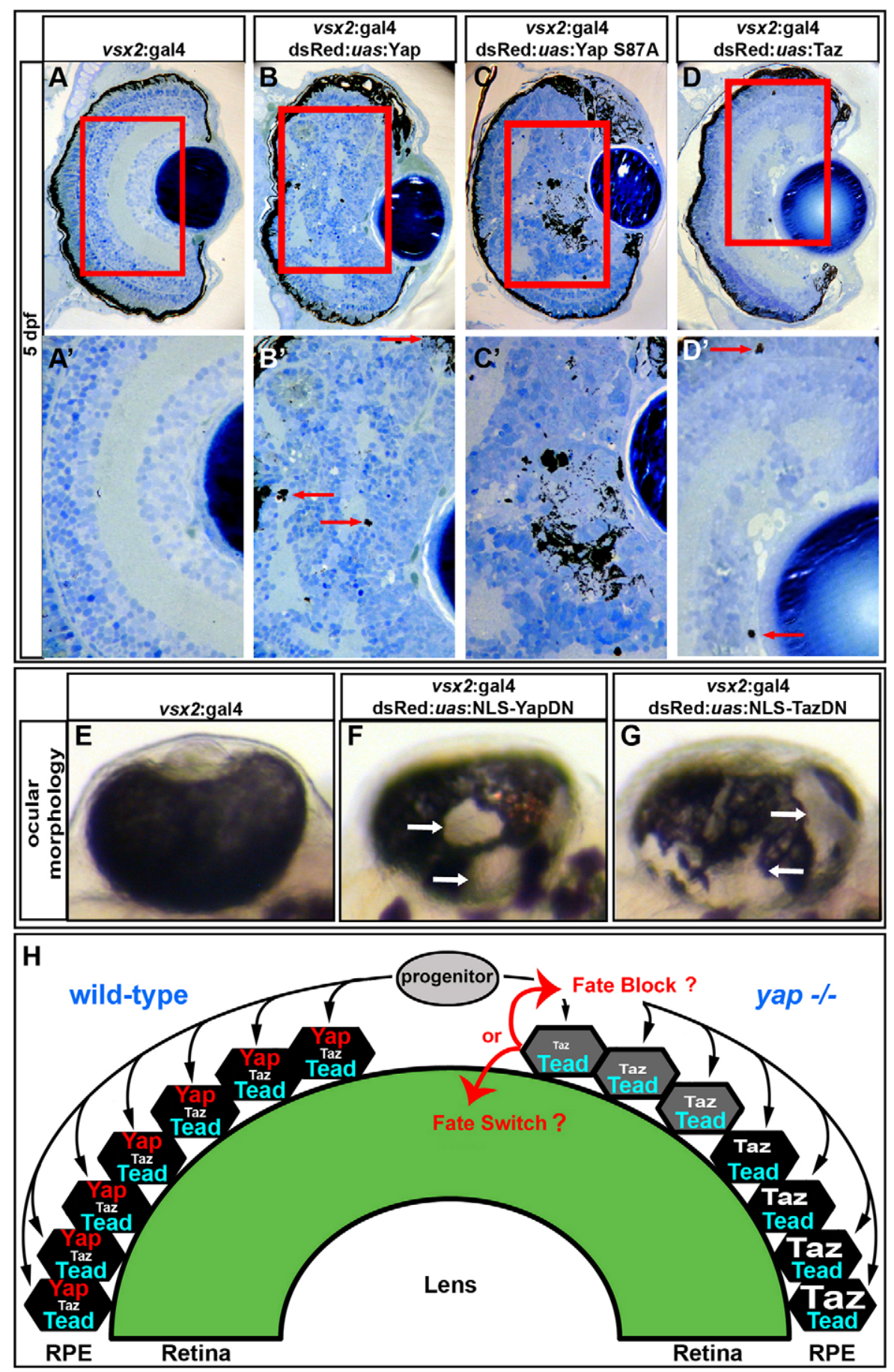

Fig. 8. Overexpression of gain- and loss-offunction yap transgenes alters RPE and NR cell fate. (A-D') Images showing that overexpression of wild-type Yap (B,B'), constitutively active Yap (Yap S87A) $\left(C, C^{\prime}\right)$ and Taz $\left(D, D^{\prime}\right)$ induces ectopic pigmentation and retinal disorganization at $5 \mathrm{dpf}$, as compared with the control $\left(A, A^{\prime}\right)$. Arrows indicate the presence of ectopic pigment cells. (E-G) Mosaic overexpression of dominant-negative forms of Yap $(\mathrm{F})$ and $\mathrm{Taz}(\mathrm{G})$ results in ectopic loss of RPE cells at $48 \mathrm{hpf}$, as compared with the control (E). Arrows indicate areas devoid of RPE cells. $(\mathrm{H})$ Model of zebrafish RPE development. Bipotent progenitor cells assume either an RPE or NR fate based on Yap/Taz activity. In the absence of yap (right hemisphere of the eye cup), progenitors contributing to the central retina cannot form RPE. Those progenitors that contribute to the peripheral eye cup can upregulate Taz during their more lengthy migration and therefore form RPE.
(Table 1). $\mathrm{yap}^{-/-} ; \mathrm{taz}^{-/-}$cells were able to form neural crest-derived melanocytes indicating that the melanization pathway is functional (data not shown). These results suggest Yap and Taz are essential for optic vesicle cells to contribute to the RPE.

Complementing the loss-of-function analyses, vsx2:Gal4-driven overexpression of wild-type Yap, constitutively active Yap (Yap S87A) and, to a lesser extent, wild-type Taz, in retinal progenitors led to ectopic pigmentation as well as disrupted lamination within the NR at $5 \mathrm{dpf}$ (Fig. 8A-D'). To determine if the morphological changes were reflected in altered retinal mRNA expression profiles we sequenced RNA from $36 \mathrm{hpf}$ eyes overexpressing Yap S87A, which generated the strongest ectopic RPE phenotype. The top 20 transcripts upregulated by Yap S87A included cyr61 and ctgf, which are established targets of Yap-Tead signaling, and both were verified as upregulated by RT-qPCR (supplementary material Table S3, Fig. S3). Other upregulated mRNA transcripts included many implicated in RPE and pigment cell genesis, differentiation and function (Table 2). Upregulation of the RPE-specific dct gene was verified by in situ hybridization in $48 \mathrm{hpf}$ retinae (supplementary material Fig. S4A-B"). Additionally, destabilized GFP expressed from a ctgf promoter transgene $(-1.0 \mathrm{~kb}$ ctgfa: d2GFP) showed increased fluorescence in RPE cells and ectopic expression in the NR (supplementary material Fig. S3B-F") in response to $v s x 2$-mediated Yap overexpression.

The vsx2:Gal4 transgene is mosaically expressed in RPE progenitor cells as well as NR progenitors (Miesfeld and Link, 
Table 1. $\mathrm{yap}^{-1-} ; \mathrm{taz}^{-1-}$ transplanted cells do not contribute to the RPE

\begin{tabular}{|c|c|c|c|c|c|}
\hline Genotype & $\mathrm{H} 2 \mathrm{~A}-\mathrm{GFP}^{+} \mathrm{NR}$ & Pigment $^{+}$RPE & Retina/RPE ratio & RPE/retina (\%) & $P$-value \\
\hline $\operatorname{yap}^{+/+} ; \operatorname{taz}^{+/+}$ & 70 & 30 & 2.3 & 42.9 & \\
\hline $\operatorname{yap}^{+/+} ; t^{\prime} z^{+/-}$ & 37 & 8 & 4.6 & 21.6 & 0.154 \\
\hline yap $^{+/+} ; t^{-1 /-}$ & 31 & 10 & 3.1 & 32.3 & 0.544 \\
\hline $\operatorname{yap}^{+/-} ; t_{2} z^{+/-}$ & 86 & 36 & 2.4 & 42.0 & 1 \\
\hline $\operatorname{yap}^{+1-} ; \operatorname{taz}^{-1-}$ & 44 & 19 & 2.3 & 43.2 & 1 \\
\hline $\operatorname{yap}^{-1-} ; \operatorname{taz}^{+/+}$ & 33 & 3 & 11 & 9.1 & 0.011 \\
\hline $\operatorname{yap}^{-1-} ; \operatorname{taz}^{+/-}$ & 47 & 9 & 5.2 & 19.1 & 0.057 \\
\hline
\end{tabular}

$P$-values were obtained using Fischer's exact comparison.

2014) and thus if exogenous Yap were to trigger the migration of existing RPE cells into the NR, this could contribute to the phenotype. However, RPE-specific $-2.7 \mathrm{~kb}$ tfec:Gal4-driven overexpression of Yap S87A did not lead to any obvious migration of Yap S87A-positive RPE cells into the NR (supplementary material Movies 5 and 6, Fig. S4C-C ${ }^{\prime \prime \prime}$ ). Altogether, these results support the idea that Yap and Taz are necessary and sufficient to promote optic cup cells to adopt RPE cell fate.

\section{DISCUSSION}

Our study suggests a model for RPE genesis in which strong YapTead transcriptional activity within bipotential optic cup progenitors drives RPE fate. Strong expression of the Yap/Taz-Tead-responsive

Table 2. Upregulated RPE/pigment-related transcripts in $36 \mathrm{hpf}$ NR overexpressing Yap S87A

\begin{tabular}{|c|c|c|}
\hline Transcript ID & Gene & Fold change \\
\hline ENSDART00000144551 & bloc1s2 & 8.18 \\
\hline ENSDART00000047272 & $c \times 43$ & 5.09 \\
\hline ENSDART00000061261 & $c \times 43$ & 3.07 \\
\hline ENSDART00000143374 & mitfa & 2.99 \\
\hline ENSDART00000030887 & slc45a2 & 2.86 \\
\hline ENSDART00000067479 & stra6 & 2.83 \\
\hline ENSDART00000104832 & hps5 & 2.75 \\
\hline ENSDART00000034248 & rab32a & 2.69 \\
\hline ENSDART00000132320 & pmelb & 2.68 \\
\hline ENSDART00000145138 & $d c t$ & 2.45 \\
\hline ENSDART00000074833 & $r \times 3$ & 2.44 \\
\hline ENSDART00000004533 & rpe65a & 2.38 \\
\hline ENSDART00000108943 & shroom2 & 2.34 \\
\hline ENSDART00000124194 & slc24a5 & 2.23 \\
\hline ENSDART00000148817 & oca2 & 2.20 \\
\hline ENSDART00000026017 & bhlhe40 & 2.11 \\
\hline ENSDART00000017153 & hps4 & 2.10 \\
\hline ENSDART00000110077 & best1 & 1.91 \\
\hline ENSDART00000006538 & otx1a & 1.85 \\
\hline ENSDART00000123568 & pmela & 1.81 \\
\hline ENSDART00000122238 & tyr & 1.73 \\
\hline ENSDART00000133864 & gpr143 & 1.69 \\
\hline ENSDART00000109822 & hps1 & 1.68 \\
\hline ENSDART00000013835 & bloc1s1 & 1.59 \\
\hline ENSDART00000059972 & crabp2a & 1.58 \\
\hline ENSDART00000039597 & $c r a b p 2 b$ & 1.57 \\
\hline ENSDART00000049863 & tfec & 1.52 \\
\hline ENSDART00000139555 & gpr143 & 1.52 \\
\hline ENSDART00000078594 & tyrp1b & 1.51 \\
\hline ENSDART00000050528 & tyrp1a & 1.43 \\
\hline ENSDART00000046268 & pmelb & 1.29 \\
\hline ENSDART00000126097 & otx2 & 1.29 \\
\hline ENSDART00000025036 & gap43 & 1.23 \\
\hline
\end{tabular}

Values indicate fold change in Yap S87A-expressing eyes compared with sibling controls. All transcripts were significantly upregulated based on an adjusted $P$-value $<0.05$.
4xGTIIC:FP transgene in presumptive RPE suggested a potential role for the downstream transcriptional components of the Hippo signaling pathway in RPE development. Although the $4 x$ GTIIC:FP transgene reports both Yap and Taz activity, Yap appears to be the main regulator of RPE cell fate. Consistent with this idea, $y a p^{-/-}$ embryos are devoid of a subset of RPE cells, whereas $t a z^{-/-}$ embryos develop normal RPE. The difference in phenotypes is consistent with the low levels of Taz expression in optic vesicle cells. Although $\mathrm{taz}^{-/-}$embryos develop normal RPE cells, compromising Taz function on a $y a p^{-/-}$background enhanced the loss of RPE cell phenotype as compared with $\mathrm{yap}^{-/-}$mutants alone. These observations place Yap and Taz as key regulators of RPE fate determination.

An interesting facet of the RPE phenotype in $\mathrm{yap}^{-/-}$embryos is the incomplete loss of RPE and the ability to rescue the phenotype through low-temperature rearing, implying that another protein, or proteins, can supplement for the loss of Yap. Based on our observations, we believe Taz is responsible for this compensation. Although the levels of taz transcript in $y a p^{-/}$embryos are not increased, the $\mathrm{yap}^{-/-}$RPE phenotype is taz gene dosage-dependent and Taz protein was elevated in $\mathrm{yap}^{-/-}$mutants. That taz mRNA levels are unaffected is consistent with observations in $y \mathrm{ap}^{-/-}$ cardiomyocytes, which also show taz gene dosage-dependent phenotypes (Xin et al., 2013). The decreased taz mRNA levels could be the result of increased nuclear localization of Taz, which negatively feeds back on taz transcription. Changes in the stability of Taz might contribute to the increased Taz protein levels accompanying decreased taz mRNA. Taz contains a phosphodegron site that is primed by Lats phosphorylation, resulting in subsequent ubiquitylation and degradation (Liu et al., 2010). Potentially, the stability of Taz could be changed by a decrease in phosphorylation of the phosphodegron in yap mutants.

The following scenario may explain the partial loss of the RPE phenotype of yap mutants. Within yap ${ }^{-/}$embryos Taz stability might be enhanced, but it might take time for translated Taz protein to reach sufficient levels for RPE specification. If so, those progenitor cells that spread and migrate further to the periphery prior to differentiation may have time to stabilize Taz and consequently generate RPE (Fig. $8 \mathrm{H}$ ). The progenitor cells in the central eye differentiate at or near their site of origin and, consequently, Taz may have insufficient time to reach the levels necessary to promote RPE fate (Fig. 8H). Because RPE and NR differentiate from the same pool of cells within the optic cup, the cells that fail to adopt RPE fate in $\mathrm{yap}^{-/-}$mutants might instead become NR progenitors, but it is also possible that these cells assume extra-ocular cell type fates (Fig. $8 \mathrm{H}$ ).

Slowing development by low-temperature rearing might allow sufficient Taz levels to accumulate, enabling compensatory Taz activity within central progenitor cells, thus explaining the rescue of 
$y a p^{-/-}$phenotypes by temperature shift. Consistent with this idea, low-temperature rearing does not rescue RPE when Taz is also abrogated. Importantly, cell transplantations indicated that $\mathrm{yap}^{-1-}$; $\mathrm{taz}^{-/-}$double-homozygous mutant cells are completely incapable of generating RPE. Similarly, dominant-negative Yap or Taz disrupts RPE genesis whereas, conversely, overexpression of either Yap or Taz can drive RPE fate from optic cup progenitor cells.

We do not know the upstream mechanism controlling Yap/Taz activity during RPE genesis, although it is likely that cross-talk with other signaling pathways is involved. WNT, BMP and TGF $\beta$ signaling all control aspects of RPE/NR development through the control of Smads and $\beta$-catenin (Sinn and Wittbrodt, 2013; Fuhrmann et al., 2014), both of which have been shown to interact with Yap or Taz (Varelas et al., 2010; Azzolin et al., 2012). Consequently, it is possible Yap/Taz-Tead activity regulates the transcription of RPE-specific genes in concert with the transcription factor targets of these pathways.

Yap and Taz have multiple binding partners within the cytoplasm and nucleus, the best-characterized of which are the Tead transcription factors. Our data imply that Yap/Taz-Tead interactions are responsible for RPE genesis. First, the $4 x G T I I C$ : FP Tead-responsive transgenic reporter is active early within RPE progenitor cells. Second, nuclear localized dominant-negative Yap and Taz proteins can cause RPE loss. Finally, loss of RPE was observed with overexpression of the Yap-binding-deficient Teadla $\mathrm{Y} 417 \mathrm{H}$ form, and within eyes in the yap Tead-binding-mutant allele. Despite the loss of RPE cells within $y a p^{\Delta T B / \triangle T B}$ mutants, the embryos maintained wild-type levels of yap mRNA and robust levels of Yap protein. Loss of Tead binding by Yap in other contexts also results in similar phenotypes to yap loss-of-function and Yapbinding-deficient alleles of TEAD1 (Fossdal et al., 2004; Williamson et al., 2014). SCRA patients carry an autosomal dominant mutation in the Yap-binding domain of TEAD1 and the loss of central RPE in these patients mimics the phenotypes observed in zebrafish $y_{a p^{-/}}$and Teadla Y417H overexpression embryos (Fossdal et al., 2004; Jonasson et al., 2007). Given these similarities, our data suggest that the congenital defects in the RPE of SCRA patients arise from autonomous Yap/Tead functional deficits of cells within the optic cup, as in yap loss-of-function zebrafish mutants.

As observed in cases of human mutations of YAPl we see phenotypic variability within and across our different zebrafish yap mutant genotypes. Notable differences occur when yap ${ }^{-/-}$ phenotypes are compared with yap ${ }^{n l 13 / n l 13}$ phenotypes. The loss of RPE is less evident in yap ${ }^{\text {nl13/nl13 }}$ mutants but there is a striking coloboma, similar to that observed in human patients with YAPI mutations (Williamson et al., 2014). We have not resolved why coloboma is evident in this mutant, but one intriguing possibility is that it could be a consequence of compromised RPE generation in the ventral retina. This might introduce mechanical disruptions to the movements of cells lining the fissure that do not occur when RPE generation is disrupted in the central retina. In this regard, it is interesting to note that recent studies of yap mutant medaka fish suggest that this pathway is a crucial regulator of actomyosin contractility (Porazinski et al., 2015). To address this issue will require manipulation of Yap/Taz-Tead activity in different populations of cells of the ventral retina to determine the cause of the colobomatous phenotype.

Our data reveal a role for Yap and Taz activity in regulating the ability of optic vesicle cells to adopt RPE cell fate. The idea that combined Yap/Taz activity is crucial for RPE fate determination is consistent with recently published reports of Yki and Yap/Taz influencing cell fate decisions in various other tissues (Nishioka et al., 2009; Zhang et al., 2011; Judson et al., 2013; Yimlamai et al., 2014). Our data potentially have implications for the efficient directed differentiation of stem cells to RPE fate - an important goal, given the initial success of stem cell-differentiated RPE cell transplants into patients with macular degeneration (Schwartz et al., 2015).

To conclude, in this study we revealed that Yap and Taz influence RPE cell fate in a gene dosage-dependent manner. The underlying mechanism is through a cell-autonomous nuclear interaction of Yap/Taz with Tead transcription factors. The elucidation of this basic mechanism offers a framework for continued investigation into the upstream and downstream protein-protein and protein-DNA interactions in eye development. The mutant lines and transgenic resources generated in this study will also facilitate resolution of the mechanistic basis of ocular phenotypes seen in patients with SCRA and congenital retinal coloboma caused by aberrant Yap/Taz-Tead activity.

\section{MATERIALS AND METHODS \\ Mutant generation}

TALENs for Yap and Taz were generated by Cellectis Bioresearch and supplied as plasmids (pTAL.CMV-T7.013535, pTAL.CMV-T7.013558, pTAL.CMV-T7.013534 and pTAL.CMV-T7.01355). The left yap TALEN arm targeted exon 1 (5'-TGAGGTTGAGAAAGCTG-3') and $15 \mathrm{nt}$ downstream in exon 1 was the right yap TALEN arm target sequence (5'TTTGGCTCTGGCGGCGT-3'). The left taz TALEN arm targeted exon 1 (5'-TGCCGCAGTCTTTCTTC-3') and 15 nt downstream in exon 1 was the right taz TALEN arm target sequence (5'-TGCCGGGAGTGGGAGCC-3'). mRNA was generated from the supplied plasmids using the mMessage mMachine and Poly(A) Tailing Kit (Ambion) and injected into 1- to 4-cell stage embryos at $150 \mathrm{pg}$ per TALEN arm. Offspring from the yap or taz TALEN-injected embryos were screened using restriction sites within each spacer region. Once founders were identified, offspring were raised and tested for allele heterozygosity. The $n l 13$ allele was identified in a forward genetic screen of fish carrying N-ethyl-N-nitrosourea-induced mutations carried out by Alex Nechiporuk's group (Oregon Health and Science University). Mapping and identification of the causative mutation followed standard procedures (Valdivia et al., 2011).

\section{Genotyping}

The Puregene Core Kit A (Qiagen) was used to extract genomic DNA (gDNA) from zebrafish tissue. Each gene region of interest was PCR amplified (supplementary material Table S4) and assessed for the presence or absence of a restriction site. The yap 4 bp deletion mutant ( $m w 48)$ was detected by the addition of a TfiI restriction site, while the yap 21 bp ( $m w 69$ ) and taz $5 \mathrm{bp}(m w 49)$ deletion mutants were detected by the loss of HinfI.

\section{Generation of plasmids and transgenic lines}

All plasmids were generated using Gateway (Invitrogen) entry clones and recombination in conjunction with the Tol2 Kit (Kwan et al., 2007). The $2.7 \mathrm{~kb}$ proximal upstream region of $t f e c$ was obtained through PCR of zebrafish gDNA and using recombination eGFP was inserted downstream of the $-2.7 k b$ tfec promoter $(-2.7 k b t f e c$ :eGFP). RT-PCR was performed to generate Flag- or Myc-tagged full-length cDNA sequences for zebrafish yap and teadla. A Tead-binding-deficient Yap (Yap S54A) was generated using QuikChange (Stratagene) site-directed mutagenesis. The Yap-bindingdeficient Teadla (Tead1a Y417H) mutant was made by including nucleotide changes within the $3^{\prime}$ PCR primer used for cloning. Flag-Yap, Flag-Yap S54A, Myc-Tead1a and Myc-Tead1a Y417H were inserted downstream of the CMV/SP6 promoter using recombination. Yap, Yap S87A, Taz, Myc-Tead1a Y417H and Yap and Taz dominant-negative constructs were generated as previously described (Miesfeld and Link, 2014). Transposase mRNA was injected with the fully assembled Tol2 constructs to generate each transgenic line (Kawakami, 2005). Transgenic and mutant lines are listed in supplementary material Table S5. 


\section{Immunofluorescence}

Standard methodology was used for whole-mount immunofluorescence and imaging (Clark et al., 2011). A 1:200 dilution of Yap rabbit polyclonal (Cell Signaling, 4912) or Taz/Yap (D24E4) rabbit monoclonal (Cell Signaling, 8418 ) and a 1:800 dilution of Alexa 488 anti-rabbit (Invitrogen) were used for Yap and Taz detection. Nuclei were labeled with TO-PRO-3 (Molecular Probes).

\section{Transfection assay}

HEK293 cells were grown in DMEM (Gibco) supplemented with 10\% FBS and penicillin and streptomycin at $37^{\circ} \mathrm{C}$ in $5 \% \mathrm{CO}_{2}$. Cells were transfected with Lipofectamine 2000 (Invitrogen) according to the manufacturer's instructions. Plasmids transfected included pTol2:CMV/SP6:Flag-Yap ${ }^{W T}$ : pA, pTol2:CMV/SP6:Flag-Yap ${ }^{S 54 A}:$ A, pTol2:CMV/SP6:Myc-Teadla ${ }^{W T}$. $p A$ and $p$ Tol2:CMV/SP6:Myc-Teadla ${ }^{Y 417 H}: p A$

\section{Immunoprecipitations and western blotting}

Cells were harvested $24 \mathrm{~h}$ after transfection in lysis buffer $(50 \mathrm{mM}$ Tris- $\mathrm{HCl}$ $\mathrm{pH} 8.0,150 \mathrm{mM} \mathrm{NaCl}, 1 \% \mathrm{NP}-40$ ) supplemented with a protease inhibitor cocktail (Roche), lysed in liquid nitrogen, centrifuged at 10,000 rpm $(11,180 \mathrm{~g})$ for $10 \mathrm{~min}$, and the supernatants used for immunoprecipitation analysis. Immunoprecipitations were performed with rabbit monoclonal anti-Myc (Cell Signaling, 2278) or rabbit monoclonal anti-Flag (Sigma-Aldrich, F7425) antibodies and protein G-sepharose beads (GE Healthcare) overnight on a rotator at $4^{\circ} \mathrm{C}$. Proteins were eluted from the beads by boiling for $5 \mathrm{~min}$ in SDS sample buffer. Elutes were fractionated by SDSPAGE (7.5\% Mini-Protean TGX, Bio-Rad) and transferred to Immobilon FL PVDF membranes (Millipore). Membranes were incubated for $1 \mathrm{~h}$ at room temperature in Odyssey blocking buffer (LI-COR Biosciences), and incubated overnight at $4^{\circ} \mathrm{C}$ with anti-Myc or anti-Flag primary antibodies diluted 1:1000 in Odyssey blocking buffer, then with near infrared fluorescent secondary antibodies (IRDye 680RD and IRDye $800 \mathrm{CW}$, LICOR Biosciences) for $1 \mathrm{~h}$ at room temperature. Proteins were detected with an Odyssey infrared imager (LI-COR Biosciences).

Equivalent numbers $(n=20)$ of $2 \mathrm{dpf}$ wild-type and $\mathrm{yap}^{-/-}$whole embryos were de-yolked (Link et al., 2006) and processed in lysis buffer and centrifuged as previously described. The supernatants were boiled for $5 \mathrm{~min}$ in SDS sample buffer and all subsequent steps were as described above. Taz was detected using a 1:1000 dilution of Taz/Yap (D24E4) rabbit monoclonal (Cell Signaling, 8418).

\section{In situ hybridization}

Embryos were fixed in 4\% paraformaldehyde at $48 \mathrm{hpf}$ and standard methodology was followed as previously described (Thisse and Thisse, 2004).

\section{Proliferation analysis}

Embryos were collected from incrosses of $y{ }^{+/}{ }^{+} ; h 2 a f z: \mathrm{GFP}^{+}$fish and raised in $0.003 \% \mathrm{~N}$-phenylthiourea (PTU) diluted in Instant Ocean fish water at $28.5^{\circ} \mathrm{C}$ before analysis, and transferred to $100 \%$ fish water after analysis. Embryos were dechorionated at 14, 18 or $24 \mathrm{hpf}$, anesthetized in 3-amino benzoic acid ethyl ester (Tricaine, Sigma-Aldrich), and mounted in 1\% lowmelt agarose (Fischer Scientific) in glass-bottom Petri dishes. Both eye fields were imaged for each embryo using confocal microscopy. After imaging, embryos were freed and raised individually in 48-well plates. The genotype of each embryo was determined by the loss of RPE phenotype at $48 \mathrm{hpf}$. Mitotic cells for each image were counted using ImageJ software (NIH). A cell was counted as mitotic if a metaphase plate was clearly visible in a single or recently divided nucleus. This same analysis was performed at $14 \mathrm{hpf}$ for $-2.7 k b$ tfec: $\mathrm{eGFP}^{+}$cells. To inhibit proliferation we treated wild-type embryos with $20 \mathrm{mM}$ hydroxyurea (Sigma-Aldrich), $150 \mu \mathrm{M}$ aphidicolin (Cayman Chemical) and 3\% DMSO in fish water from 10.5-26 hpf.

\section{Cell death analysis}

Embryos were incubated at $28.5^{\circ} \mathrm{C}$ for $20 \mathrm{~min}$ in $20 \mathrm{ml} 5 \mu \mathrm{g} / \mathrm{ml}$ Acridine Orange (Sigma-Aldrich) diluted in Instant Ocean embryo media at 14, 18 or $24 \mathrm{hpf}$. After incubation, embryos were briefly washed four times in PTU, anesthetized in Tricaine and embedded in 1\% low-melt agarose in glass- bottom Petri dishes and imaged by confocal microscopy. Embryos were freed and reared individually in a 48-well plate. Genotypes were assessed based on the loss of RPE phenotype. Cell death counts were performed using MetaMorph (Molecular Devices). Cell death was inhibited by coinjecting $150 \mu \mathrm{M}$ tp53 morpholino (Robu et al., 2007) and $100 \mathrm{pg} \mathrm{bcl-xl}$ mRNA (Sidi et al., 2008) into 1- to 2-cell stage embryos.

\section{Quantitative real-time PCR analysis}

All cDNA was generated using the Superscript III First-Strand Synthesis System for RT-PCR Kit (Invitrogen) per manufacturer's instructions and all qRT-PCR was performed on a CFX96 and CFX Connect Real-Time System (Bio-Rad) using SsoAdvanced SYBR Green Supermix (Bio-Rad). Analysis of yap and taz transcripts was performed on whole embryos. mRNA was extracted from dechorionated wild-type (ZDR), $\mathrm{yap}^{-/-}$and $y a p^{\Delta T B / \Delta T B}$ embryos at $32 \mathrm{hpf}$ using the RNeasy Plus Mini Kit (Qiagen) per manufacturer's instructions. qRT-PCR was performed using equivalent amounts of mRNA for four biological replicates of ten pooled embryos for ZDR and $\mathrm{yap}^{-/-}$embryos, while three biological replicates of ten pooled embryos were used for $y a p^{\Delta T B / \Delta T B}$ embryos. All biological replicates were run in triplicate for each transcript. The zebrafish housekeeping gene efla was used for normalization.

For verification of transcripts identified as upregulated in RNA-seq, whole eye tissue from $36 \mathrm{hpf}$ embryos expressing Yap S87A or nonexpressing siblings was pooled ( 15 embryos/30 eyes) and mRNA was extracted using TRIzol (Invitrogen). All transcripts were measured in triplicate for each of three independent biological replicates analyzed. efla was used for normalization.

\section{Blastulae transplantation}

Donor cells were transplanted and targeted into host embryos as previously described (Kemp et al., 2009). After transplantation, donor embryos were genotyped and host embryos raised individually in 48-well plates containing fish water. Retinal clones were scored between 1 and $2 \mathrm{dpf}$ based on the presence of $\mathrm{eGFP}^{+}$nuclei. RPE clones were assessed at $2 \mathrm{dpf}$ based on pigmentation and the characteristic hexagonal shape of RPE cells (Fig. 7). Only eyes that contained $\mathrm{H} 2 \mathrm{~A}-\mathrm{GFP}^{+} \mathrm{NR}$ clones were assessed for RPE clones. Fischer's exact test was used to compare the RPE/NR ratio of each mutant genotype combination with the wild-type ratio.

\section{RNA-seq}

Yap S87A and sibling control whole eyes were dissected at $36 \mathrm{hpf}$ and immediately frozen on dry ice until $\sim 60$ pooled retinas were obtained for each genotype. RNA was purified as described (Uribe et al., 2012), except that RNA was eluted in a $50 \mu$ final volume. RNA quality was determined using an Agilent BioAnalyzer. 50 bp single-read sequencing was performed in triplicate for each genotype using an Illumina HiSeq2000 at VANTAGE (Vanderbilt University). Sequencing results were analyzed by VANGARD (Vanderbilt University). RNA-seq reads were mapped to D. rerio cDNA sequences from Ensembl release 66. RNA-seq data have been deposited at GEO under accession GSE71681.

\section{Acknowledgements}

We thank Jon Bostrom, Peter Volberding and Rod Young for assistance with molecular biology; Emily Seminary for assistance with supplementary material Fig. S3; Pat Cliff, Bill Hudzinski and the UCL Fish Facility team for zebrafish husbandry; Dr James Lister for the dct in situ probe plasmid; Dr Kristen Kwan and Dr Masa Tada for the $b c l-x l$ and yap-GFP mRNA plasmids, respectively; and Alex Nechiporuk for kindly providing the n/13 allele for analysis.

\section{Competing interests}

The authors declare no competing or financial interests.

\section{Author contributions}

J.B.M., G.G., J.C.B., S.W.W. and B.A.L. designed experiments. J.B.M. and B.A.L. co-wrote the paper with help from G.G. and S.W.W. J.B.M., B.S.C. and M.A.F. conducted experiments for RNA-seq and validation. R.J.P. performed sequence analysis to identify the $n / 13$ mutation. J.R.B. and J.B.M. conducted the immunoprecipitation assays and G.G. performed all experiments using the n/13 allele. All authors helped edit the manuscript. 


\section{Funding}

RNA-seq experiments were performed in VANTAGE (Vanderbilt University, Nashville, TN, USA), which is supported by the Vanderbilt Ingram Cancer Center [P30 CA68485], the Vanderbilt Vision Center [P30 EY08126] and National Institutes of Health/National Center for Research Resources (NIH/NCRR) [G20 RR030956]. This project was supported by an $\mathrm{NIH} /$ National Eye Institute (NEI) training grant [T32EY014536 to J.B.M. and B.S.C.] to the Medical College of Wisconsin (MCW) vision research community; an NIH grant [R01EY014167] to B.A.L.; a pilot project grant from the MCW Cancer Center - Advancing a Healthier Wisconsin (to B.A.L.) an NEI core facilities grant [P30EY001931] to the vision research community of MCW; UK Medical Research Council and Wellcome Trust grants to S.W.W. and G.G.; and a Wellcome Trust career development fellowship to R.J.P. Deposited in PMC for immediate release.

\section{Supplementary material}

Supplementary material available online at

http://dev.biologists.org/lookup/suppl/doi:10.1242/dev.119008//DC1

\section{References}

Azzolin, L., Zanconato, F., Bresolin, S., Forcato, M., Basso, G., Bicciato, S., Cordenonsi, M. and Piccolo, S. (2012). Role of TAZ as mediator of Wnt signaling. Cell 151, 1443-1456.

Cao, X., Pfaff, S. L. and Gage, F. H. (2008). YAP regulates neural progenitor cell number via the TEA domain transcription factor. Genes Dev. 22, 3320-3334

Chen, L., Chan, S. W., Zhang, X., Walsh, M., Lim, C. J., Hong, W. and Song, H. (2010). Structural basis of YAP recognition by TEAD4 in the hippo pathway. Genes Dev. 24, 290-300.

Clark, B. S., Winter, M., Cohen, A. R. and Link, B. A. (2011). Generation of Rabbased transgenic lines for in vivo studies of endosome biology in zebrafish. Dev Dyn. 240, 2452-2465.

Fossdal, R., Jonasson, F., Kristjansdottir, G. T., Kong, A., Stefansson, H., Gosh, S., Gulcher, J. R. and Stefansson, K. (2004). A novel TEAD1 mutation is the causative allele in Sveinsson's chorioretinal atrophy (helicoid peripapillary chorioretinal degeneration). Hum. Mol. Genet. 13, 975-981.

Fuhrmann, S., Zou, C. and Levine, E. M. (2014). Retinal pigment epithelium development, plasticity, and tissue homeostasis. Exp. Eye Res. 123, 141-150.

Heermann, S., Schütz, L., Lemke, S., Krieglstein, K. and Wittbrodt, J. (2015) Eye morphogenesis driven by epithelial flow into the optic cup facilitated by modulation of bone morphogenetic protein. eLife 4, e05216.

Jonasson, F., Hardarson, S., Olafsson, B. M. and Klintworth, G. K. (2007) Sveinsson chorioretinal atrophy/helicoid peripapillary chorioretinal degeneration: first histopathology report. Ophthalmology 114, 1541-1546.

Judson, R. N., Tremblay, A. M., Knopp, P., White, R. B., Urcia, R., De Bari, C., Zammit, P. S., Camargo, F. D. and Wackerhage, H. (2013). The Hippo pathway member Yap plays a key role in influencing fate decisions in muscle satellite cells. J. Cell Sci. 125, 6009-19.

Kawakami, K. (2005). Transposon tools and methods in zebrafish. Dev. Dyn. 234 244-254.

Kemp, H. A., Carmany-Rampey, A. and Moens, C. (2009). Generating chimeric zebrafish embryos by transplantation. J. Vis. Exp. 29, e1394.

Kitagawa, M. (2007). A Sveinsson's chorioretinal atrophy-associated missense mutation in mouse Tead1 affects its interaction with the co-factors YAP and TAZ. Biochem. Biophys. Res. Commun. 361, 1022-1026.

Kwan, K. M., Fujimoto, E., Grabher, C., Mangum, B. D., Hardy, M. E., Campbell, D. S., Parant, J. M., Yost, H. J., Kanki, J. P. and Chien, C.-B. (2007). The Tol2kit: a multisite gateway-based construction kit for Tol2 transposon transgenesis constructs. Dev. Dyn. 236, 3088-3099.

Kwan, K. M., Otsuna, H., Kidokoro, H., Carney, K. R., Saijoh, Y. and Chien, C.-B. (2012). A complex choreography of cell movements shapes the vertebrate eye. Development 139, 359-372.

Link, V., Shevchenko, A. and Heisenberg, C.-P. (2006). Proteomics of early zebrafish embryos. BMC Dev. Biol. 6, 1

Lister, J. A., Lane, B. M., Nguyen, A. and Lunney, K. (2011). Embryonic expression of zebrafish MiT family genes tfe3b, tfeb, and tfec. Dev. Dyn. 240 2529-2538.

Liu, C.-Y., Zha, Z-Y., Zhou, X., Zhang, H., Huang, W., Zhao, D., Li, T., Chan, S. W., Lim, C. J., Hong, W. et al. (2010). The hippo tumor pathway promotes TAZ degradation by phosphorylating a phosphodegron and recruiting the SCF $\{$ beta\}TrCP E3 ligase. J. Biol. Chem. 285, 37159-37169.

Mann, C. J., Osborn, D. P. S. and Hughes, S. M. (2007). Vestigial-like-2b (VITO1b) and Tead-3a (Tef-5a) expression in zebrafish skeletal muscle, brain and notochord. Gene Expr. Patterns 7, 827-836.
Miesfeld, J. B. and Link, B. A. (2014). Establishment of transgenic lines to monitor and manipulate Yap/Taz-Tead activity in zebrafish reveals both evolutionarily conserved and divergent functions of the Hippo pathway. Mech. Dev. 133 177-188

Naye, F., Treguer, K., Soulet, F., Faucheux, C., Fedou, S., Theze, N. and Thiebaud, P. (2007). Differential expression of two TEF-1 (TEAD) genes during Xenopus laevis development and in response to inducing factors. Int. J. Dev. Biol. 51, 745-752.

Nishioka, N., Inoue, K.-i., Adachi, K., Kiyonari, H., Ota, M., Ralston, A., Yabuta, N., Hirahara, S., Stephenson, R. O. and Ogonuki, N. (2009). The Hippo signaling pathway components Lats and Yap pattern Tead4 activity to distinguish mouse trophectoderm from inner cell mass. Dev. Cell 16, 398-410.

Picker, A., Cavodeassi, F., Machate, A., Bernauer, S., Hans, S., Abe, G., Kawakami, K., Wilson, S. W. and Brand, M. (2009). Dynamic coupling of pattern formation and morphogenesis in the developing vertebrate retina. PLoS Biol. 7 e1000214.

Porazinski, S., Wang, H., Asaoka, Y., Behrndt, M., Miyamoto, T., Morita, H. Hata, S., Sasaki, T., Krens, S. F., Osada, Y. et al. (2015). YAP is essential for tissue tension to ensure vertebrate 3D body shape. Nature 521, 217-221.

Robu, M. E., Larson, J. D., Nasevicius, A., Beiraghi, S., Brenner, C., Farber S. A. and Ekker, S. C. (2007). p53 activation by knockdown technologies. PLoS Genet. 3, e78.

Schwartz, S. D., Regillo, C. D., Lam, B. L., Eliott, D., Rosenfeld, P. J., Gregori, N. Z., Hubschman, J.-P., Davis, J. L., Heilwell, G., Spirn, M. et al. (2015). Human embryonic stem cell-derived retinal pigment epithelium in patients with age-related macular degeneration and Stargardt's macular dystrophy: follow-up of two open-label phase 1/2 studies. Lancet 385, 509-516.

Sidi, S., Sanda, T., Kennedy, R. D., Hagen, A. T., Jette, C. A., Hoffmans, R., Pascual, J., Imamura, S., Kishi, S., Amatruda, J. F. et al. (2008). Chk1 suppresses a caspase-2 apoptotic response to DNA damage that bypasses p53 $\mathrm{Bcl}-2$, and caspase-3. Cell 133, 864-877.

Sinn, R. and Wittbrodt, J. (2013). An eye on eye development. Mech. Dev. 130 347-358.

Streisinger, G., Singer, F., Walker, C., Knauber, D. and Dower, N. (1986) Segregation analyses and gene-centromere distances in zebrafish. Genetics 112 311-319.

Thisse, B. and Thisse, C. (2004). High throughput expression analysis in zebrafish ZFIN direct submission, https://zfin.org/ZDB-PUB-040907-1.

Tsetskhladze, Z. R., Canfield, V. A., Ang, K. C., Wentzel, S. M., Reid, K. P., Berg A. S., Johnson, S. L., Kawakami, K. and Cheng, K. C. (2012). Functional assessment of human coding mutations affecting skin pigmentation using zebrafish. PLoS ONE 7, e47398.

Uribe, R. A., Kwon, T., Marcotte, E. M. and Gross, J. M. (2012). Id2a functions to limit Notch pathway activity and thereby influence the transition from proliferation to differentiation of retinoblasts during zebrafish retinogenesis. Dev. Biol. 371 280-292.

Valdivia, L. E., Young, R. M., Hawkins, T. A., Stickney, H. L., Cavodeassi, F. Schwarz, Q., Pullin, L. M., Villegas, R., Moro, E., Argenton, F. et al. (2011). Lef1-dependent Wnt/beta-catenin signalling drives the proliferative engine that maintains tissue homeostasis during lateral line development. Development 138 3931-3941.

Varelas, X. (2014). The Hippo pathway effectors TAZ and YAP in development, homeostasis and disease. Development 141, 1614-1626.

Varelas, X., Samavarchi-Tehrani, P., Narimatsu, M., Weiss, A., Cockburn, K., Larsen, B. G., Rossant, J. and Wrana, J. L. (2010). The Crumbs complex couples cell density sensing to Hippo-dependent control of the TGF-beta-SMAD pathway. Dev. Cell 19, 831-844.

Williamson, K. A., Rainger, J., Floyd, J. A. B., Ansari, M., Meynert, A., Aldridge, K. V., Rainger, J. K., Anderson, C. A., Moore, A. T., Hurles, M. E. et al. (2014). Heterozygous loss-of-function mutations in YAP1 cause both isolated and syndromic optic fissure closure defects. Am. J. Hum. Genet. 94, 295-302.

Xin, M., Kim, Y., Sutherland, L. B., Murakami, M., Qi, X., McAnally, J., Porrello, E. R., Mahmoud, A. I., Tan, W., Shelton, J. M. et al. (2013). Hippo pathway effector Yap promotes cardiac regeneration. Proc. Natl. Acad. Sci. USA 110 13839-13844.

Yimlamai, D., Christodoulou, C., Galli, G. G., Yanger, K., Pepe-Mooney, B. Gurung, B., Shrestha, K., Cahan, P., Stanger, B. Z. and Camargo, F. D. (2014) Hippo pathway activity influences liver cell fate. Cell 157, 1324-1338.

Zhang, T., Zhou, Q. and Pignoni, F. (2011). Yki/YAP, Sd/TEAD and Hth/MEIS control tissue specification in the Drosophila eye disc epithelium. PLoS ONE 6, e22278.

Zhao, B., Ye, X., Yu, J., Li, L., Li, W., Li, S., Lin, J. D., Wang, C.-Y., Chinnaiyan, A. M., Lai, Z.-C. et al. (2008). TEAD mediates YAP-dependent gene induction and growth control. Genes Dev. 22, 1962-1971. 


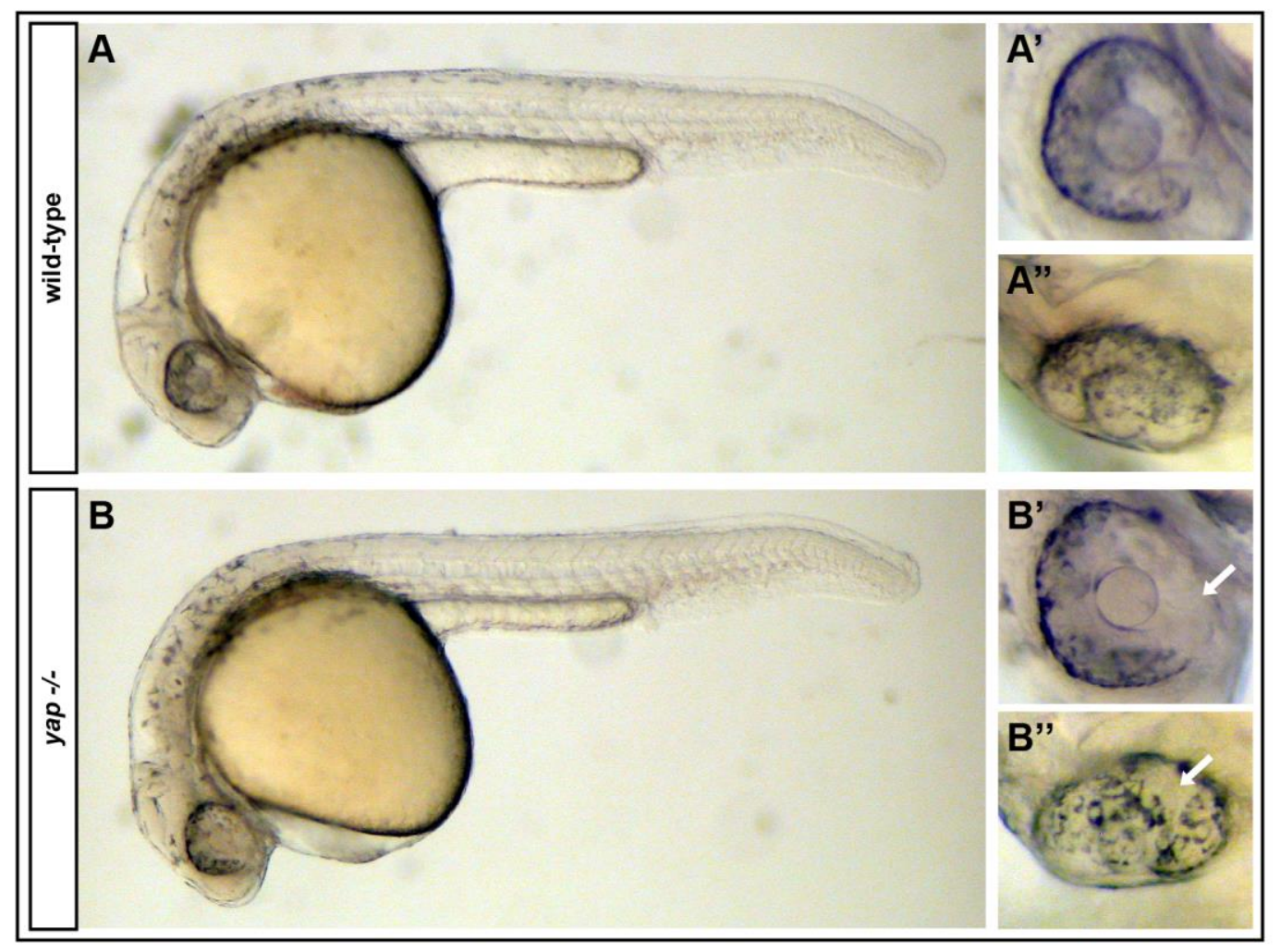

Figure S1: yap $^{-/}$mutants exhibit RPE defects noticeable at the onset of pigmentation.

(A-A') A Wild-type embryo with normal RPE cells and pigmentation encompassing the whole eye globe.

(B-B') $y^{-/-}$embryos lack RPE cells before RPE cells are completely pigmented. White arrows $=$ areas of absent of RPE cells. 


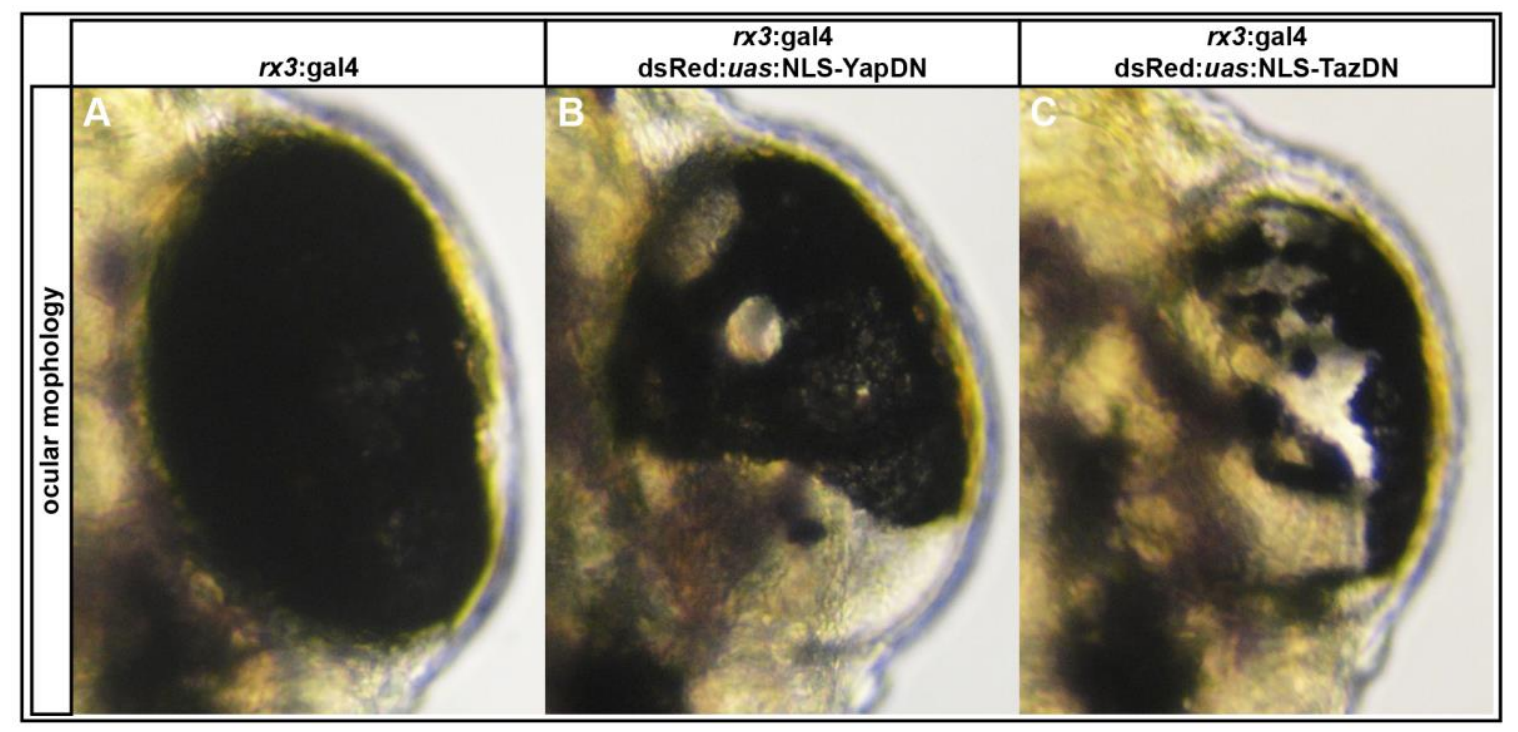

Figure S2: Overexpression of NLS-YapDN and NLS-TazDN in $r x 3$ positive cells results in loss of RPE.

(A-C) Examples of eyes from $r x 3:$ gal4+/dsRed:uas:NLS-YapDN and rx3:gal4+/dsRed:uas:NLS-TazDN fry lacking RPE cells at $48 \mathrm{hpf}$. 


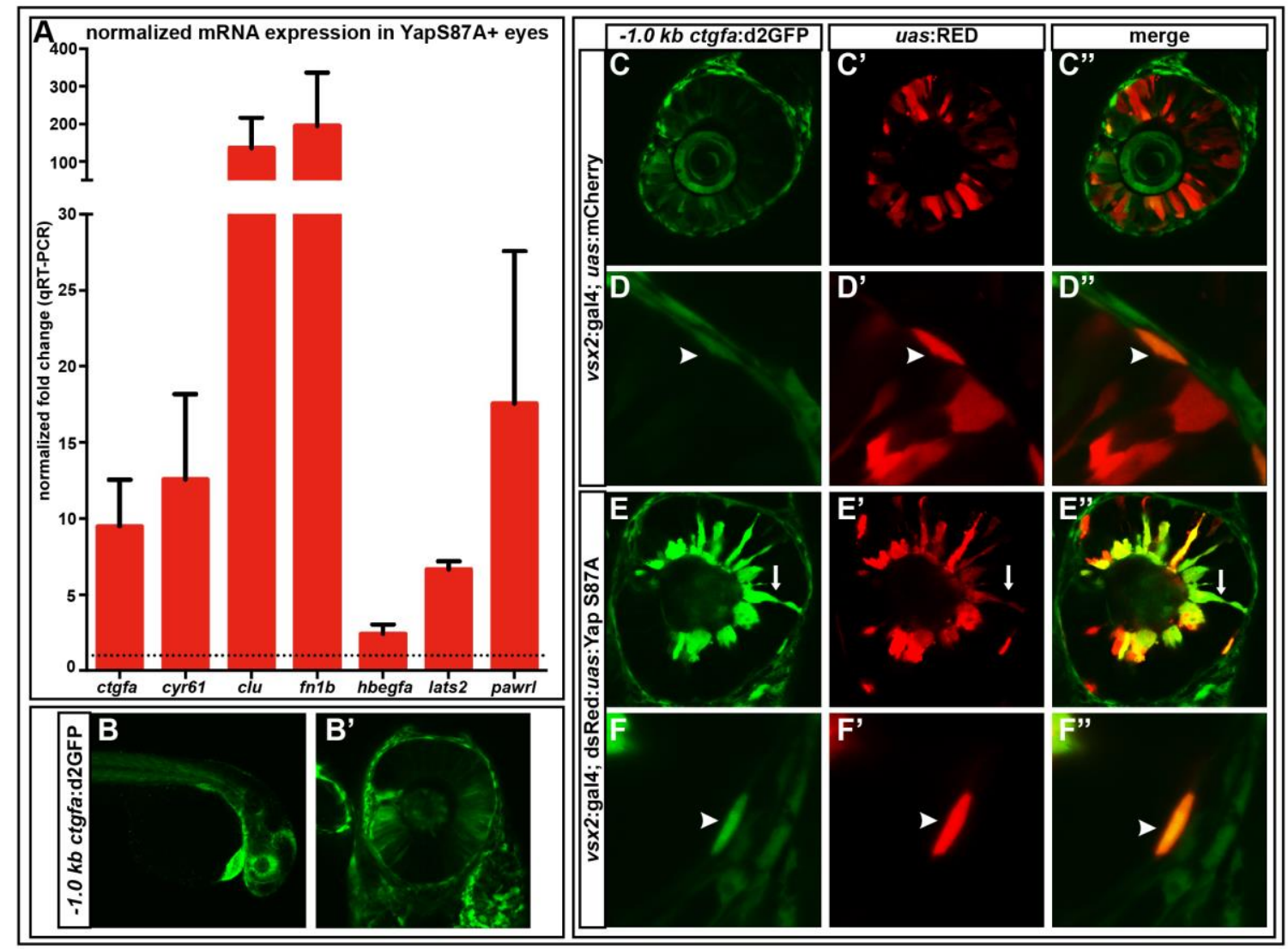

Figure S3: qRT-PCR validates mRNA transcripts that were upregulated via RNAseq in Yap S87A overexpressing $36 \mathrm{hpf}$ eyes and $-1.0 \mathrm{~kb}$ ctgfa:d2GFP is upregulated by Yap S87A.

(A) ctgfa (9.5-fold, $p=0.0508)$, cyr61 (12.6-fold, $p=0.1072)$, clu (135.5-fold, $p=0.1705)$, fn $1 b$ (195-fold, $p=0.2418)$, hbegfa ( 2.4 -fold, $p=0.0845)$, lats 2 (6.7-fold, $p=0.0005$ ), pawrl (17.6-fold, $p=0.1736$ ). Dashed line indicates the normalized expression levels of yap and taz in wild-type embryos. An unpaired t-test was performed on mRNA expression and statistical significance was performed using the Holm-Sidak method. Error bars = s.e.m. (B-B') $-1.0 \mathrm{~kb}$ ctgfa: $\mathrm{d} 2 \mathrm{GFP}$ expression is observed in the NR, RPE, heart, and other tissues at $28 \mathrm{hpf}$. (C-F') Yap S87A overexpression results in increased -1.0kb ctgfa:d2GFP in RPE cells (F-F'") and ectopic NR expression (E-E') compared to mCherry (C-D'). Arrows=ectopic NR, arrow heads=RPE expression. All embryos are 28 hpf. 

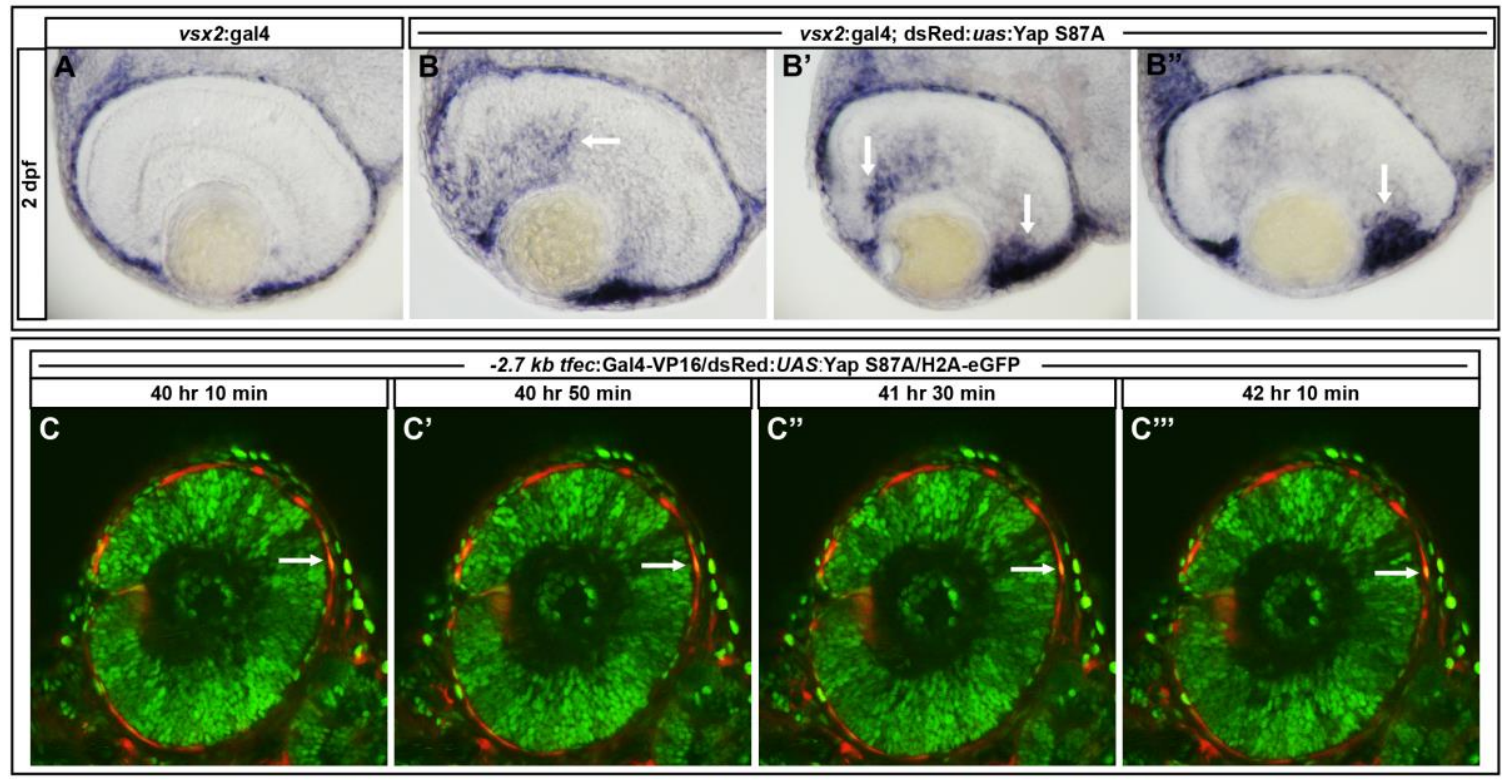

Figure S4: Overexpression of Yap S87A in vsx2 positive cells results in ectopic expression of $d c t$ mRNA and $-2.7 \mathrm{~kb}$ tfec:Gal4-VP16/dsRed: $U A S$ :Yap S87A positive cells do not migrate into the neural retina.

(A) Endogenous dct expression is observed in the RPE.

(B-B') Ectopic $d c t$ expression is observed in the neural retina and enhanced in the ciliary marginal zone in Yap S87A overexpressing embryos. Black arrows denote ectopic $d c t$.

(C-C',') RPE cells overexpressing Yap S87A do not ectopically migrate into the neural retina. Images represent a 2 hour time course from 40-42 hpf. White arrows indicate a Yap S87A positive RPE cell. 


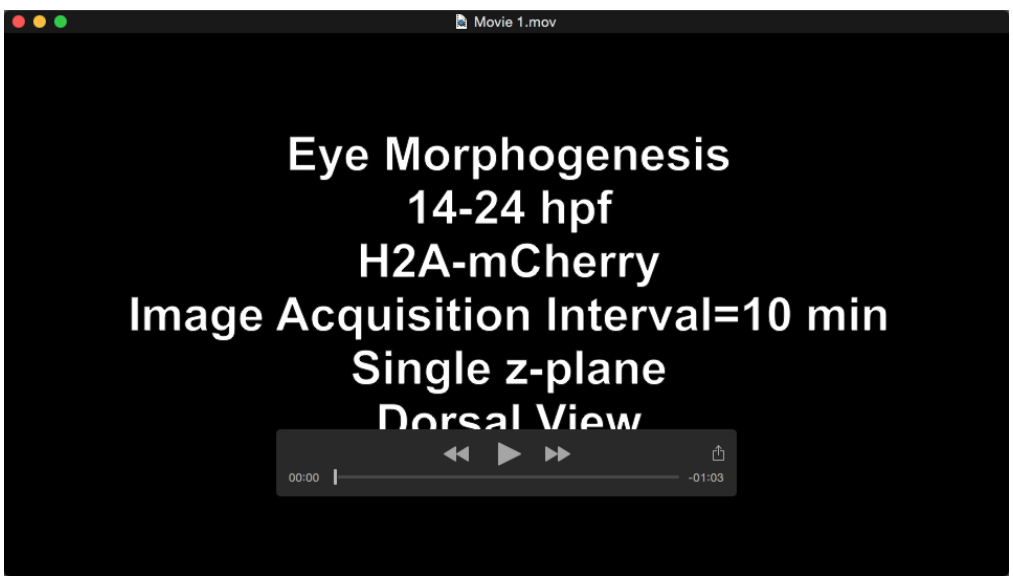

Movie 1: Time lapse of zebrafish eye morphogenesis including various transcription factors and signaling pathways involved.

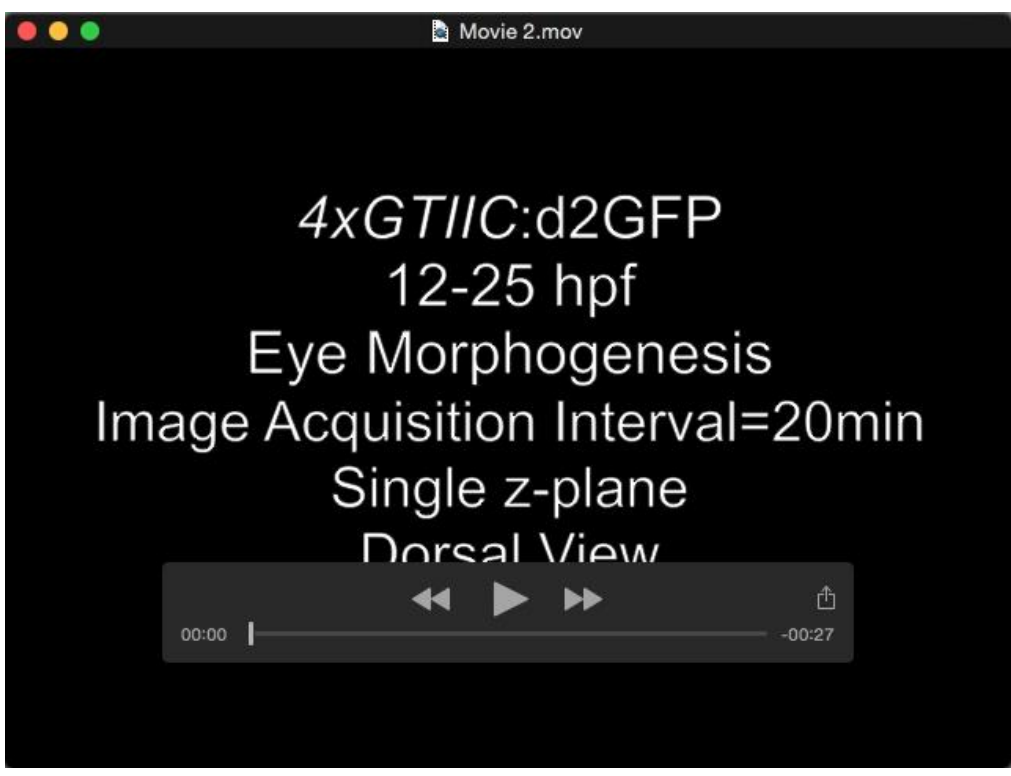

Movie 2: $4 x G T I I C$ :d2GFP transgene expression during optic cup morphogenesis. Arrows indicate RPE and lens expression. $\mathrm{L}=$ lens, $\mathrm{NR}=$ neural retina, $\mathrm{RPE}=$ retina pigment epithelium. 


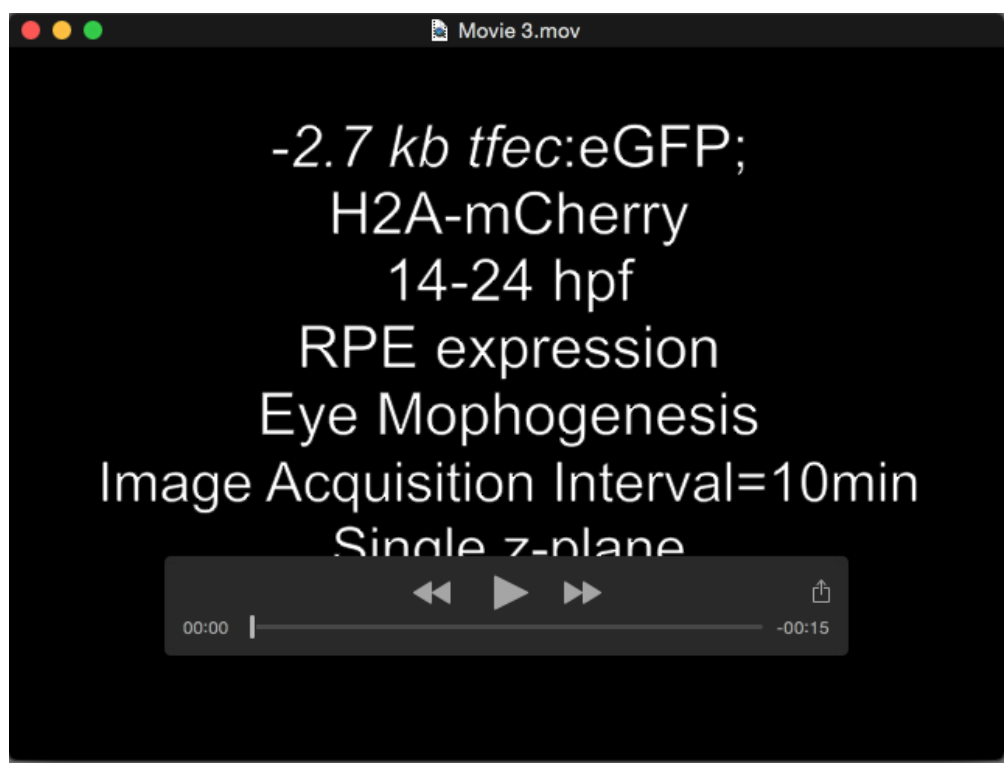

Movie 3: -2.7kb tfec:eGFP/h2afz:H2A-mCherry transgene expression from 14-24 hpf during optic cup morphogenesis.

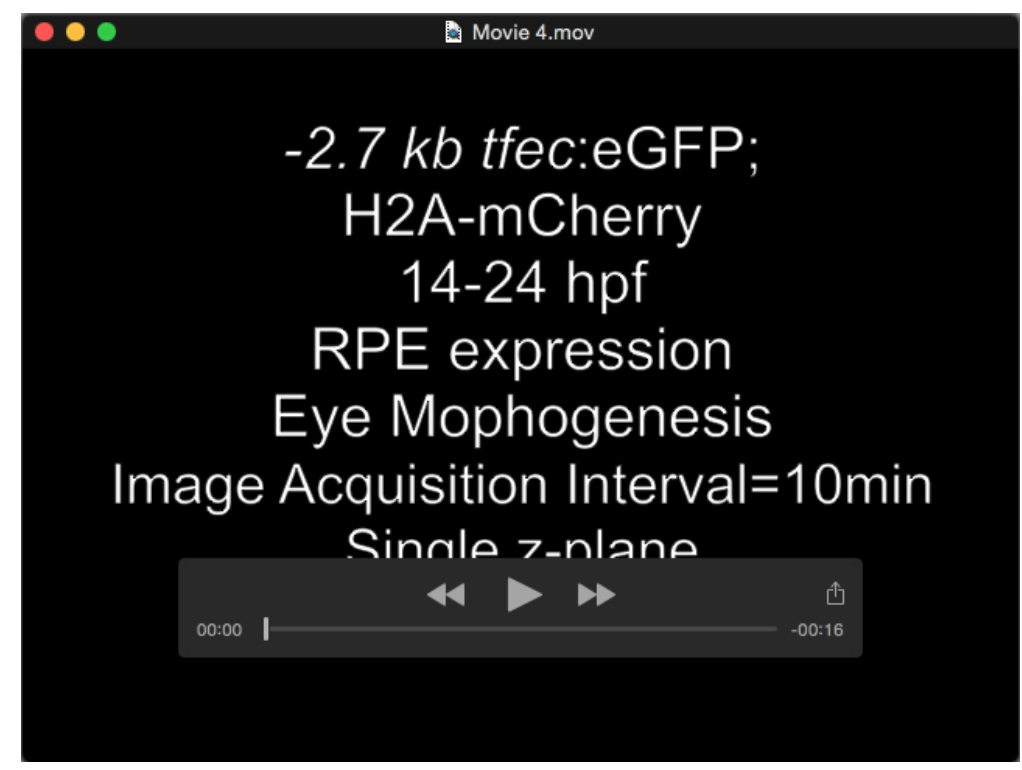

Movie 4: -2.7kb tfec:eGFP/h2afz:H2A-mCherry expression from 14-24 hpf during optic cup morphogenesis. The arrow represents a $-2.7 \mathrm{~kb}$ tfec:eGFP+ mitotic cell. 


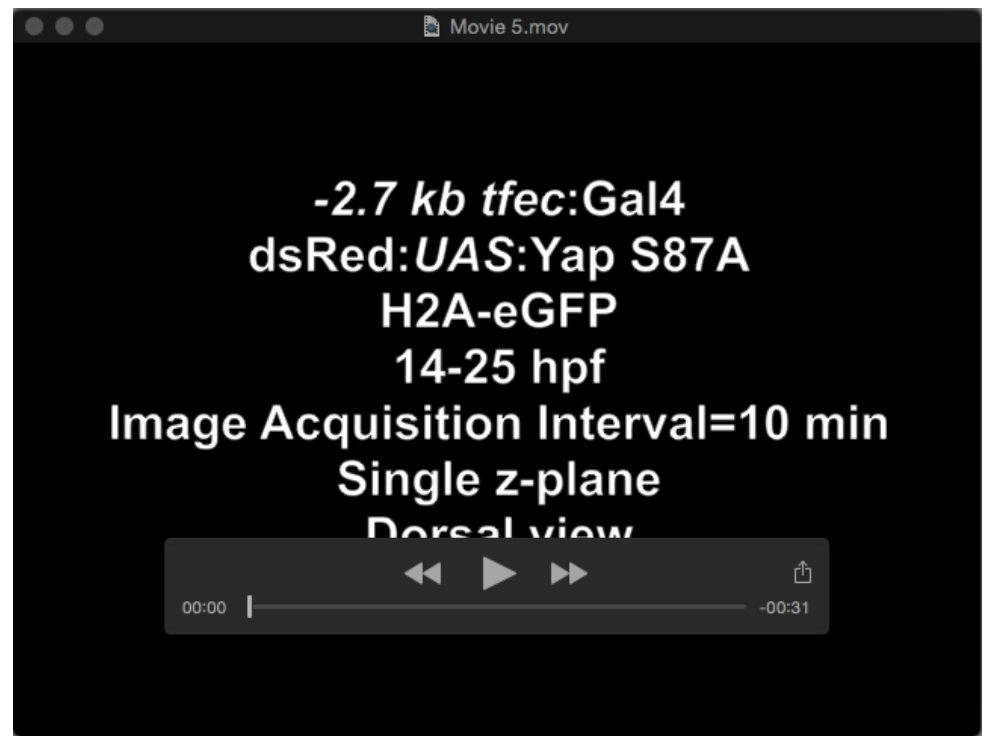

Movie 5: -2.7kb tfec:Gal4-VP16/dsRed:UAS:Yap S87A/H2A-eGFP expression from 14-25 hpf during optic cup morphogenesis. The arrows highlight $-2.7 \mathrm{~kb}$ tfec:Gal4VP16/dsRed:UAS:Yap S87A positive cells that migrating normally around the rim of the optic cup and not into the presumptive neural retina. No abnormal RPE cell migrations were noted. The time lapse movie is played twice. 


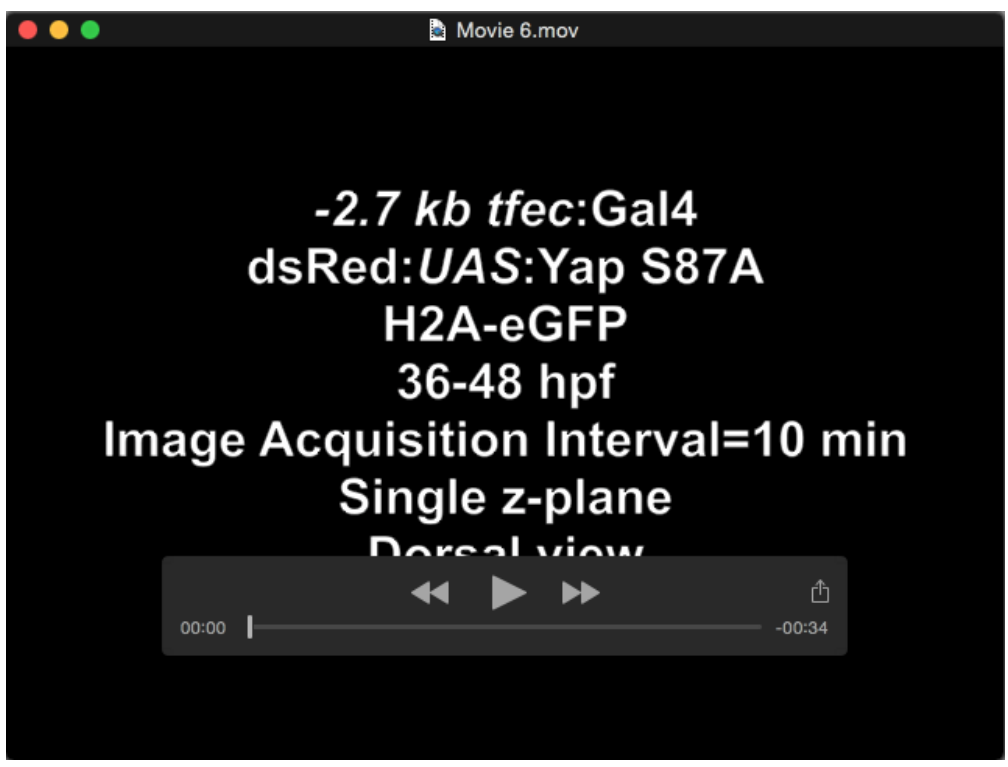

Movie 6: -2.7kb tfec:Gal4-VP16/dsRed:UAS:Yap S87A/H2A-eGFP expression from 36-48 hpf during retinal neurogenesis. The arrows highlight $-2.7 \mathrm{~kb}$ tfec:Gal4VP16/dsRed:UAS:Yap S87A positive cells that maintain their position in the RPE and do not migrate into the neural retina. No abnormal RPE cell migration was noted. The time lapse movie is played twice. 
Table S1: The loss of RPE phenotype observed in yap $^{-/}$embryos is rescued when embryos are reared at $20.5^{\circ} \mathrm{C}$. Embryos were placed at $20.5^{\circ} \mathrm{C}$ at $70 \%$ epiboly and put back at $28.5^{\circ} \mathrm{C}$ at prim-10.

\begin{tabular}{|c|c|c|}
\hline \multicolumn{3}{|c|}{ yap ${ }^{+/-} X y a p^{+/-}$} \\
\hline $28.5^{\circ} \mathrm{C}$ & $\begin{array}{c}\text { Predicted } \\
\text { Values }\end{array}$ & $\begin{array}{l}\text { Actual } \\
\text { Values }\end{array}$ \\
\hline Total Embryos Scored & 389 & 389 \\
\hline Wild Type RPE & 292 & 307 \\
\hline Mutant RPE & 97 & 82 \\
\hline$\%$ of Mutant/Wild Type RPE & $25.00 \%$ & $21 \%$ \\
\hline $20.5^{\circ} \mathrm{C}$ & $\begin{array}{c}\text { Predicted } \\
\text { Values }\end{array}$ & $\begin{array}{l}\text { Actual } \\
\text { Values }\end{array}$ \\
\hline Total Embryos Scored & 389 & 389 \\
\hline Wild Type RPE & 292 & 389 \\
\hline Mutant RPE & 97 & $\mathbf{0}$ \\
\hline$\%$ of Mutant/Wild Type RPE & $25 \%$ & $0 \%$ \\
\hline
\end{tabular}


Table S2: The loss of RPE phenotype cannot be completely rescued by $20.5^{\circ} \mathrm{C}$ rearing when a mutant $t a z$ allele is present in the a $^{-/}$background. Embryos were placed at $20.5^{\circ} \mathrm{C}$ at $70 \%$ epiboly and put back at $28.5^{\circ} \mathrm{C}$ at prim- 10 .

\begin{tabular}{|c|c|c|}
\hline \multicolumn{3}{|c|}{ 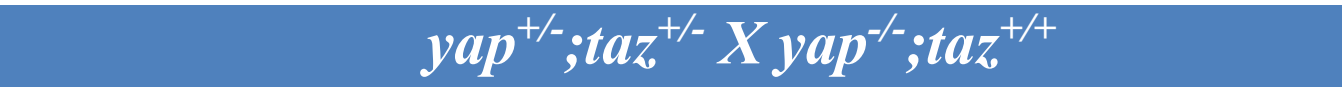 } \\
\hline $28.5^{\circ} \mathrm{C}$ & $\begin{array}{c}\text { Predicted } \\
\text { Values }\end{array}$ & $\begin{array}{l}\text { Actual } \\
\text { Values }\end{array}$ \\
\hline Total Embryos Scored & 136 & 136 \\
\hline Wild Type RPE & 68 & 74 \\
\hline Mutant RPE & 68 & 62 \\
\hline$\%$ of Mutant/Wild Type RPE & $50 \%$ & $46 \%$ \\
\hline $20.5^{\circ} \mathrm{C}$ & $\begin{array}{c}\text { Predicted } \\
\text { Values }\end{array}$ & $\begin{array}{l}\text { Actual } \\
\text { Values }\end{array}$ \\
\hline Total Embryos Scored & 135 & 135 \\
\hline Wild Type RPE & 68 & 110 \\
\hline Mutant RPE & 67 & 25 \\
\hline \% of Mutant/Wild Type RPE & $50 \%$ & $19 \%$ \\
\hline
\end{tabular}


Table S3: The top 20 most upregulated transcripts in Yap S87A overexpressing 36 hpf eyes. Values represent the fold change of Yap S87A expressing eyes compared to sibling controls. All transcripts were significantly upregulated based on an adjusted $p$ value $<0.05$

\begin{tabular}{|c|c|c|}
\hline Transcript ID & Gene & $\begin{array}{c}\text { Fold } \\
\text { Change }\end{array}$ \\
\hline ENSDART00000060765 & $B X 323876.3$ & 187.12 \\
\hline ENSDART00000141193 & clu & 179.31 \\
\hline ENSDART00000109972 & $B X 248501.1$ & 91.70 \\
\hline ENSDART00000129496 & cyr61 & 33.07 \\
\hline ENSDART00000018117 & ppp1r14aa & 20.69 \\
\hline ENSDART00000037904 & $\operatorname{socs} 3 b$ & 19.77 \\
\hline ENSDART00000109138 & hbegfa & 19.59 \\
\hline ENSDART00000104965 & plcxd1 (3 of 5) & 18.04 \\
\hline ENSDART00000003505 & adm (1 of 2) & 17.12 \\
\hline ENSDART00000112226 & apcdd1l & 16.35 \\
\hline ENSDART00000077951 & pcolce $2 b$ & 15.92 \\
\hline ENSDART00000017312 & $f n 1 b$ & 15.27 \\
\hline ENSDART00000097460 & hmgcra & 15.23 \\
\hline ENSDART00000148845 & DKEY-6N3.3 & 12.97 \\
\hline ENSDART00000063028 & $\operatorname{ctg} f$ & 12.50 \\
\hline ENSDART00000112243 & crlf1a & 11.36 \\
\hline ENSDART00000124465 & junbl & 10.79 \\
\hline ENSDART00000150088 & DKEY-119G10.4 & 10.53 \\
\hline ENSDART00000106488 & plod2 & 10.18 \\
\hline ENSDART00000145103 & cntfr & 9.91 \\
\hline
\end{tabular}


Table S4: List of primers used for genotyping and qRT-PCR. E=Efficiency \% of qRT-PCR primers.

\begin{tabular}{|c|c|c|}
\hline Primer Name & Primer Sequence & Assay \\
\hline yap $4 \mathrm{bp} / 21 \mathrm{bp} \mathrm{F}$ & $5^{\prime}$-AGTCATGGATCCGAACCAGCACAA-3' & genotyping \\
\hline yap $4 \mathrm{bp} / 21 \mathrm{bp} \mathrm{R}$ & $5^{\prime}$-TGCAATCGGCCTTTATTTTCCTGC-3' & genotyping \\
\hline $\operatorname{taz} 5 \mathrm{bp} \mathrm{F}$ & $5^{\prime}$-CTCGGCTGAAACTACTTAAGGACG-3' & genotyping \\
\hline $\operatorname{taz} 5 \mathrm{bp} \mathrm{R}$ & $5^{\prime}$-CTAAACAGTGTGCAGGAATGTCC-3' & genotyping \\
\hline yap qPCR F & $5^{\prime}$-CCAGACAAGCCAGTACAGAT-3' & $\begin{array}{l}\text { RT-qPCR } \\
\mathrm{E}=95 \%, \mathrm{r}^{2}=.997\end{array}$ \\
\hline yap qPCR R & $5^{\prime}$-GAAGTATCTCTGTCCCGAAGG-3' & $\begin{array}{l}\text { RT-qPCR } \\
\mathrm{E}=95 \%, \mathrm{r}^{2}=.997\end{array}$ \\
\hline taz qPCR F & $5^{\prime}-$ GCATCCAGATGGAGAGAGAG-3' & $\begin{array}{l}\text { RT-qPCR } \\
E=105 \%, r^{2}=.991\end{array}$ \\
\hline taz qPCR R & $5^{\prime}$-GCTGTTATTGGGCATGTTTC-3' & $\begin{array}{l}\text { RT-qPCR } \\
E=105 \%, r^{2}=.991\end{array}$ \\
\hline efl $\alpha$ exon $1-2 \mathrm{~F}$ & $5^{\prime}$ - TCTCTCAATCTTGAAACTTATCAATCA-3' & $\begin{array}{l}\text { RT-qPCR } \\
\mathrm{E}=102.7 \%, \\
\mathrm{r}^{2}=.992\end{array}$ \\
\hline efl $\alpha$ exon3 R & $5^{\prime}-\mathrm{AACACCCAGGCGTACTTGAA-3^{ \prime }}$ & $\begin{array}{l}\text { RT-qPCR } \\
\mathrm{E}=102.7 \%, \\
\mathrm{r}^{2}=.992\end{array}$ \\
\hline $\operatorname{ctg} f a \mathrm{~F}$ & $5^{\prime}-\mathrm{CTGCACAGCCAGAGATG-3^{ \prime }}$ & $\begin{array}{l}\text { RT-qPCR } \\
\mathrm{E}=120 \%, \mathrm{r}^{2}=.990\end{array}$ \\
\hline $\operatorname{ctg} f a \mathrm{R}$ & $5^{\prime}$-CACTTCCCAGGCACTTT-3' & $\begin{array}{l}\text { RT-qPCR } \\
E=120 \%, r^{2}=.990\end{array}$ \\
\hline cyr61 F & $5^{\prime}-$ CCGTGTCCACATGTACATGGG-3' & $\begin{array}{l}\text { RT-qPCR } \\
\mathrm{E}=107.7 \%, \\
\mathrm{r}^{2}=.987\end{array}$ \\
\hline cyr61 R & $5^{\prime}$-GGTGCATGAAAGAAGCTCGTC-3' & $\begin{array}{l}\text { RT-qPCR } \\
\mathrm{E}=107.7 \%, \\
\mathrm{r}^{2}=.987\end{array}$ \\
\hline $\operatorname{clu} \mathrm{F}$ & $5^{\prime}-$ GTCGCAAGTTGGTGAGAAATACC-3' & $\begin{array}{l}\text { RT-qPCR } \\
\mathrm{E}=98.7 \%, \mathrm{r}^{2}=.900\end{array}$ \\
\hline clu $\mathrm{R}$ & $5^{\prime \prime}$-СTCCTTCATCTCCTGAGCCATC-3' & $\begin{array}{l}\text { RT-qPCR } \\
\mathrm{E}=98.7 \%, \mathrm{r}^{2}=.900\end{array}$ \\
\hline$f n l b \mathrm{~F}$ & $5^{\prime \prime}$-CAGTACTGTACAGTCAGGGGAAGC-3' & $\begin{array}{l}\text { RT-qPCR } \\
\mathrm{E}=94.7 \%, \mathrm{r}^{2}=.970\end{array}$ \\
\hline$f n 1 b \mathrm{R}$ & $5^{\prime}$-CACGACCGTTGTCATTACAGCC-3' & $\begin{array}{l}\text { RT-qPCR } \\
E=94.7 \%, r^{2}=.970\end{array}$ \\
\hline hbegfa $\mathrm{F}$ & $5^{\prime}$ - CAAGCAAGGTGCATATAATGTGG-3' & $\begin{array}{l}\text { RT-qPCR } \\
E=105.6 \%, \\
r^{2}=.980\end{array}$ \\
\hline hbegfa $\mathrm{R}$ & $5^{\prime}$-CTGCCAAACAAACACGGTCAC-3' & $\begin{array}{l}\text { RT-qPCR } \\
\mathrm{E}=105.6 \%,\end{array}$ \\
\hline
\end{tabular}


Development 142: doi:10.1242/dev.119008: Supplementary Material

\begin{tabular}{|l|l|l|}
\hline & & $\mathrm{r}^{2}=.980$ \\
\hline lats $2 \mathrm{~F}$ & $5^{\prime}$-CTCCGAGAGATCCGCAAGTC-3' & $\mathrm{RT}-\mathrm{qPCR}$ \\
& & $\mathrm{E}=93 \%, \mathrm{r}^{2}=.974$ \\
\hline lats2 $\mathrm{R}$ & $5^{\prime}$-CACGTACAATCTGTTCAGTGTG-3' & $\mathrm{RT}-\mathrm{qPCR}$ \\
& & $\mathrm{E}=93 \%, \mathrm{r}^{2}=.974$ \\
\hline pawrl $\mathrm{F}$ & $5^{\prime}$-GAACAAGACCTTGCTGAAAGTG-3' & $\mathrm{RT}-\mathrm{qPCR}$ \\
& & $\mathrm{E}=104 \%, \mathrm{r}^{2}=.906$ \\
\hline pawrl $\mathrm{R}$ & $5^{\prime}$-CACTTCCACAATCCAAAGCGTCC-3' & $\mathrm{RT}-\mathrm{qPCR}$ \\
& & $\mathrm{E}=104 \%, \mathrm{r}^{2}=.906$ \\
\hline
\end{tabular}


Table S5. Transgenic and mutant zebrafish lines

\begin{tabular}{|c|c|}
\hline Line & Reference \\
\hline $\operatorname{Tg}(4 x G T I I C: \mathrm{d} 2 \mathrm{GFP})^{\mathrm{mw} 50}$ & Miesfeld and Link, 2014 \\
\hline $\operatorname{Tg}(\mathrm{dsRed} . \mathrm{T} 4: 14 x U A S: Y a p)^{\mathrm{mw} 63}$ & Miesfeld and Link, 2014 \\
\hline Tg(dsRed.T4:14xUAS:Wwtr1) ${ }^{\mathrm{mw} 64}$ & Miesfeld and Link, 2014 \\
\hline Tg(dsRed.T4:14xUAS:YapCA(S87A)) ${ }^{\mathrm{mw} 65}$ & Miesfeld and Link, 2014 \\
\hline $\operatorname{Tg}\left(\mathrm{dsRed} . \mathrm{T} 4: 14 x U A S: \mathrm{NLS}-\mathrm{Yap}^{\mathrm{DN}}\right)^{\mathrm{mw} 66}$ & This study \\
\hline $\operatorname{Tg}\left(\text { dsRed.T4:14xUAS:NLS-Taz }{ }^{\mathrm{DN}}\right)^{\mathrm{mw} 67}$ & This study \\
\hline $\operatorname{Tg}(-2.7 k b \text { tfec: } \mathrm{eGFP})^{\mathrm{mw} 68}$ & This study \\
\hline Tg(dsRed.T4:14xUAS:Myc-TeadY417H) ${ }^{\mathrm{mw} 70}$ & This study \\
\hline $\operatorname{Tg}\left(-2.7 k b\right.$ tfec:Gal4-VP16) ${ }^{\mathrm{mw} 71}$ & This study \\
\hline $\operatorname{Tg}(-5.0 \text { kb fox } C 1 b: \text { Gal4-VP16 })^{\mathrm{mw} 72}$ & This study \\
\hline $\operatorname{Tg}(-1.0 k b \text { ctgfa: } \mathrm{d} 2 \mathrm{GFP})^{\mathrm{mw} 75}$ & This study \\
\hline $\operatorname{Tg}(v s \times 2: G a 14-V P 16)^{\mathrm{mw} 39}$ & Clark et al., 2011 \\
\hline yap c. $158 \_161 d e l^{m w 48}$ & This study \\
\hline yap c.158_178del ${ }^{m w 69}$ & This study \\
\hline$w w t r 1$ c.156_160del ${ }^{m w 49}$ & taz mutants (this study) \\
\hline$y a p^{\mathrm{nl13}}$ & This study \\
\hline $\operatorname{Tg}(h 2 a f v: \mathrm{h} 2 \mathrm{afv}-\mathrm{GFP})^{\mathrm{kca} 6}$ & Pauls et al., 2001 \\
\hline $\operatorname{Tg}(\text { Ola.Rx3:Gal4-VP16) })^{\mathrm{vu} 271}$ & Weiss et al., 2012 \\
\hline albino $^{b 4}$, slc $45 a 2^{b 4 / b 4}$ & Streisinger et al., 1986, Tsetskhladze et al., 2012 \\
\hline
\end{tabular}




\section{Supplementary references}

Pauls, S., Geldmacher-Voss, B. and Campos-Ortega, J. A. (2001). A zebrafish histone variant H2A.F/Z and a transgenic H2A.F/Z:GFP fusion protein for in vivo studies of embryonic development. Dev. Genes Evol. 211, 603-610.

Weiss, O., Kaufman, R., Michaeli, N. and Inbal, A. (2012). Abnormal vasculature interferes with optic fissure closure in 1mo2 mutant zebrafish embryos. Dev. Biol. 369, 191-198. 